2

8 Sciences, ${ }^{3}$ Department of Medicine, School of Medicine, UConn Health, Farmington, CT 06030,

9 USA. $10{ }^{4}$ School of integrative Medicine, Tianjin University of Traditional Chinese Medicine, Tianjin 11301617 , China.

$12{ }^{5}$ Department of Basic Sciences, School of Medicine, Tsinghua University, Beijing, China.

$13{ }^{6}$ Section of Infectious Diseases, Yale University School of Medicine, 333 Cedar Street, New

$13{ }^{6}$ Section of Infectious D

15

16

17

18

19

20

21

22 Running title: UBXN3B controls B lymphopoiesis

\section{An Essential Role of UBXN3B in B Lymphopoiesis}

Tingting Geng ${ }^{1 *}$, Duomeng Yang ${ }^{1 *}$, Tao Lin ${ }^{1}$, Andrew G. Harrison ${ }^{1}$, Binsheng Wang ${ }^{2}$, Blake Torrance ${ }^{1}$, Kepeng Wang ${ }^{1}$, Yanlin Wang ${ }^{3}$, Long Yang ${ }^{4}$, Laura Haynes ${ }^{1}$, Gong Cheng ${ }^{5}$, Anthony T. Vella ${ }^{1}$, Erol Fikrig ${ }^{6}$ and Penghua Wang ${ }^{1 \#}$

${ }^{1}$ Department of Immunology, ${ }^{2}$ Center on Aging and Department of Genetics and Genome 301617, China.

* T.G. and D.Y contributed equally.

\# Address correspondence to: Penghua Wang, Ph.D., Department of Immunology, School of 9 Medicine, University of Connecticut, UConn Health, Farmington, CT 06030, USA. Email:

pewang@uchc.edu, Tel: 860-679-6393. 


\section{ABSTRACT}

Hematopoiesis is finely regulated to enable timely production of the right numbers and types of mature immune cells to maintain tissue homeostasis. Dysregulated hematopoiesis may compromise antiviral immunity and/or exacerbate immunopathogenesis. Herein, we report an essential role of UBXN3B in maintenance of hematopoietic homeostasis and restriction of

31 immunopathogenesis during respiratory viral infection. Ubxn3b deficient $\left(U b \times n 3 b^{-/-}\right)$mice are

32 highly vulnerable to SARS-CoV-2 and influenza A infection, characterized by more severe lung

33 immunopathology, lower virus-specific IgG, significantly fewer B cells, but more myeloid cells

34 than $U b x n 3 b^{+/+}$littermates. This aberrant immune compartmentalization is recapitulated in

35 uninfected Ubxn3b-/- mice. Mechanistically, UBXN3B controls precursor B-I (pre-BI) transition to pre-BII and subsequent proliferation in a cell-intrinsic manner, by maintaining BLNK protein

37 stability and pre-BCR signaling. These results reveal an essential role of UBXN3B for the early 38 stage of B cell development. 


\section{INTRODUCTION}

The immune system is comprised of various cell types that coordinate responses to infection, maintain tissue and immune homeostasis. Peripheral immune cells, with the exception of a few cell types such as long-lived memory $T$ cells and some tissue macrophages, are constantly replenished from bone marrow stem cells through progenitor cells ${ }^{1}$. For instance, approximately $0.5-1 \times 10^{11}$ granulocytes are generated daily in adult human individuals ${ }^{2}$. The hematopoietic system is a hierarchically organized, somatic stem cell-maintained organ system, with long-lived and self-renewing pluripotent hematopoietic stem cells (LT-HSCs) at its apex ${ }^{1}$. LT-HSCs differentiate into short-term multipotent progenitors (MPPs or ST-HSCs) and lineage-committed hematopoietic progenitors, which in turn will eventually differentiate into the numerous mature blood cell lineages ${ }^{3}$. While at the apex of the hematopoietic hierarchy, LT-HSCs are largely quiescent, and the highly proliferative MPPs are the primary contributor to steady-state hematopoiesis $^{4}{ }^{5}$. MPPs are capable of differentiating into lineage-committed progenitors, e.g., common lymphoid progenitors (CLPS) and common myeloid progenitors (CMP), which turn into blast cells leading to specific cell types. Among hematopoiesis, B cell development is the best studied, with several stages clearly defined, including pre-progenitor (pre-pro) B, precursor B I (pre-BI), large pre-BII, small pre-BII and immature B (imm-B) inside bone marrow. In bone marrow, pre-BI transition to large pre-BII is considered an essential checkpoint, involving rearrangement of variable (V)/diversity (D)/joining $(J)$ gene segments by recombination activating genes (RAG1/2) and assembly of pre-B cell receptor (pre-BCR) with a surrogate light chain (SLC) and immunoglobulin $(\mathrm{lg}) \mu$ heavy chain $(\mu \mathrm{H})$ on cell surface. Pre-BI cells without a functional pre-BCR undergo apoptosis. Once passing the first quality check, pre-BI becomes large pre-BII, which proliferates several rounds and turn into small pre-BII. At this stage, small 
67

pre-BII no longer expresses SLC, but begins expressing an lg light chain $\mathrm{k}$ or $\lambda$ that forms a BCR together with $\mu \mathrm{H}$, and becomes imm-B (checkpoint 2). Imm-B cells exit bone marrow and mature in the peripheral immune organs such as spleen and lymph node ${ }^{6}$. This process is controlled by a unique set of cell-intrinsic transcription factors and cell-extrinsic factors such as cytokines, chemokines and growth factors in its bone marrow niche ${ }^{3}$.

The human genome encodes 13 ubiquitin regulatory $X(U B X)$ domain-containing proteins, designated UBXNs. The UBX domain shares weak homology with ubiquitin but adopts the same three dimensional fold as ubiquitin ${ }^{7}$. Many UBXNs are capable of binding multiple E3 ubiquitin ligases and p97 (also known as VCP), an ATPase associated with various cellular activities (AAA ATPase) ${ }^{8,9}$. However, the physiological functions of UBXNs remain poorly characterized. We and other research groups have recently shown that several UBXNs regulate viral RNAsensing RIG-I (retinoic acid inducible gene 1) like receptor -mitochondrial antiviral viral signaling (RLR-MAVS) ${ }^{10-12}$ and Nuclear factor-kB (NF-kB) signaling pathways ${ }^{13,14}$. Of note, we recently reported that UBXN3B controls a DNA virus infection by positively regulating the dsDNAsensing cGAS (cyclic di-GMP-AMP synthase)-STING (stimulator-of-interferon-genes) signaling and innate immunity ${ }^{15}$. However, the physiological function of UBXN3B in RNA virus infection remains unknown. To this end, we will study two important respiratory viruses, including one positive-sense single-stranded RNA [(+) ssRNA] severe acute respiratory syndrome (COVID19)-causing coronavirus 2 (SARS-CoV-2), and a negative-sense single-stranded RNA [(-) ssRNA] influenza A virus (IAV). Both viruses induce life-threatening lung immunopathology, typified by elevated levels of inflammatory mediators, myeloid immune infiltrates in the lung, neutrophilia and lymphopenia ${ }^{16} 17$. Herein, we report that $U b x n 3 b$ deficient $\left(U b x n 3 b^{-/-}\right)$mice are 
highly vulnerable to SARS-CoV-2 and IAV infection, typified by higher viral loads and

91 inflammatory mediators, more severe immunopathology in the lung, but lower virus-specific

92 immunoglobulin (lg) $\mathrm{G}$ and slower resolution of disease, when compared to Ubxn $3^{+/+}$littermates.

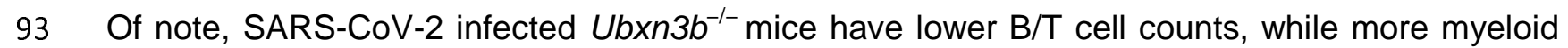

94 cells, and consequently a higher neutrophil-to-lymphocyte ratio (N/L) in the lung and blood.

95 Intriguingly, this abnormal immune compartmentalization is also recapitulated in uninfected

$96 U b \times n 3 b^{-/-}$mice when compared to $U b \times n 3 b^{+/+}$littermates. Reciprocal bone marrow

97 transplantation reveals that the $\mathrm{B}$ cell defect in $U b \times n 3 b^{-/}$is cell-intrinsic. Mechanistically,

98 UBXN3B controls precursor B-I (pre-BI) transition to pre-BII and subsequent proliferation in a 99 cell-intrinsic manner, by maintaining BLNK protein stability and pre-BCR signaling.

100

101

\section{RESULTS}

102

103

\section{UBXN3B restricts pathogenesis of respiratory viruses}

104 We have long been interested in UBXNs because of their potential function in ubiquitination and 105 immune regulation. Using a tamoxifen-inducible Cre-LoxP system, we recently successfully 106 deleted an essential gene, UBXN3B, in adult mice and demonstrated that UBXN3B positively 107 regulates the STING-mediated type I IFN response to a DNA virus ${ }^{15}$. Because STING signaling 108 also plays a role in controlling some RNA viruses in an IFN-dependent or -independent manner, 109 we continued investigating the physiological role of UBXN3B in restricting RNA virus infection. 110 To this end, we tested with respiratory viruses of public health significance including SARS111 CoV-2 and influenza A virus (IAV). Because mice are barely permissive to clinical isolates of 112 SARS-CoV-2, we delivered human angiotensin-converting enzyme 2 (ACE2, the cellular entry 
receptor for SARS-CoV)-expressing Ad5 vector (replication-defective adenovirus vector)

114 intranasally to the mouse lung before infection ${ }^{18}{ }^{19}$. We observed a slight drop in body mass a

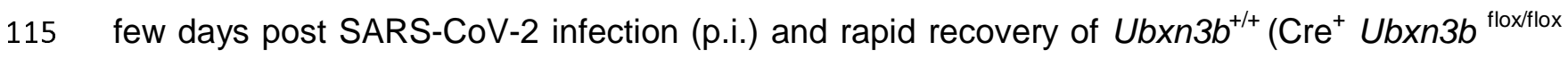

116 treated with corn oil) mice, while a $\sim 10 \%$ reduction in the body weight of $U b \times n 3 b^{--}\left(\mathrm{Cre}^{+} \mathrm{Ubxn3b}\right.$

117 flox/flox treated with tamoxifen dissolved in corn oil) littermates by days 3-4 p.i. and a significant

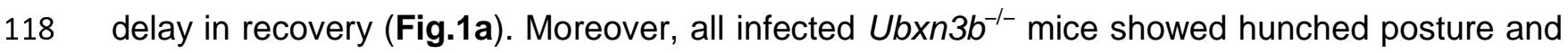

119 decreased mobility at day 2 p.i., while $U b \times n 3 b^{+/+}$animals behaved normally (Suppl Movie 1 and

120 2). Histopathological analyses by hematoxylin and eosin (H\&E) staining revealed more immune

121 infiltrates in the lung of both $U b x n 3 b^{+/+}$and $U b x n 3 b^{-/-}$mice at day 3 p.i., compared to uninfected

122 mice (day 0). However, there was no significant difference in the numbers of immune infiltrates

123 between the two groups (Fig.1b). At day 10 p.i., many clusters of brownish cells were noted in

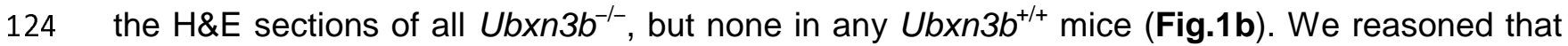

125 these brownish cells were representative of hemosiderosis, a form of iron overload disorder

126 resulting in the accumulation of hemosiderin, an iron-storage complex. In the lung,

127 macrophages phagocytose red blood cells due to vascular leakage, leading to iron overload.

128 Using iron staining we tested this hypothesis and detected a few lightly iron-laden cells at day 3

129 p.i., but many clusters of heavily iron-laden cells by day 10 p.i. in all the $U b x n 3 b^{-/-}$lungs

130 compared to $U b x n 3 b^{+/+}$(Fig.1c, d). On day 35 p.i., we still noted moderate lung hemosiderosis

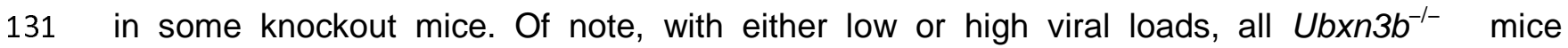

132 presented a similar degree of hemosiderosis at days 3 and 10 p.i., while no $U b \times n 3 b^{+/+}$mouse

133 had it (Fig. 1c, d), suggesting that the severe lung damage in Ubxn3b-/- mice is primarily

134 caused by immunopathology. We next asked if these observations could be extended to other

135 respiratory viruses, such as influenza. Indeed, Ubxn3 $3 b^{-/-}$mice lost more body weight; and of 
136

137

138

note, $70 \%$ of them succumbed to a dose of $\mathrm{H} 1 \mathrm{~N} 1$ influenza $A$ that was only sublethal to $U b \times n 3 b^{+/+}$mice (Fig.1e, f).

Severe COVID-19 pathogenesis is a combination of a direct cytopathic effect of SARS-CoV-2 replication and hyper-inflammation in the lung ${ }^{16}$. In particular, COVID-19 fatality is strongly associated with elevated inflammatory mediators such interleukin 6 (IL-6) and tumor necrosis factor $(T N F-a)$ etc. ${ }^{16}$. We first examined viral loads and immune gene expression. The viral loads in lungs trended higher in $U b \times n 3 b^{-/-}$when compared to $U b \times n 3 b^{+/+}$mice, though they varied significantly among individuals at day 3 p.i. By day 10 p.i. the virus was almost cleared from the lung in both mouse genotypes (Suppl Fig.s1a). The serum cytokines IL-6, TNF-a, IL-10

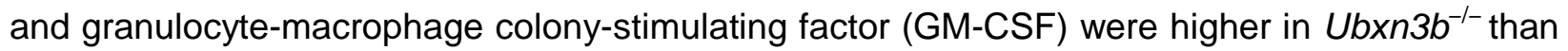
those in $U b x n 3 b^{+/+}$on day 3 p.i. (Suppl Fig.s1b), which is consistent with clinical observations in severe COVID-19 patients. The concentrations of serum interferon alpha (IFN- $\alpha$ ), C-X-C motif chemokine ligand $10(\mathrm{CXCL} 10)$ and IFN-Y were modestly upregulated, but equally between $U_{b} \times n 3 b^{-/-}$and $U b \times n 3 b^{+/+}$mice after SARS-CoV-2 infection, suggesting a normal type I/II IFN response in Ubxn3b-/- (Suppl Fig.s1b).

\section{UBXN3B maintains immune homeostasis during SARS-CoV-2 infection}

COVID-19 fatality is strongly associated with an imbalance in immune cell compartmentalization, characterized by neutrophilia and lymphopenia ${ }^{16}$. We thus analyzed neutrophils and $\mathrm{T}$ cells in the lung by flow cytometry and found that the total CD45 ${ }^{+}$immune cell counts per lung were 10-fold higher in SARS-CoV-2-infected animals than mock-treated animals. However, there was no significant difference between $U b \times n 3 b^{-/-}$and $U b x n 3 b^{+/+}$littermates (Fig.2a). Upon close 


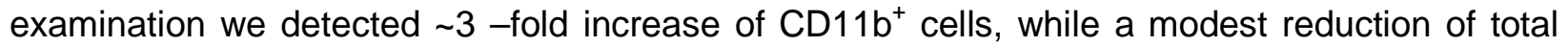

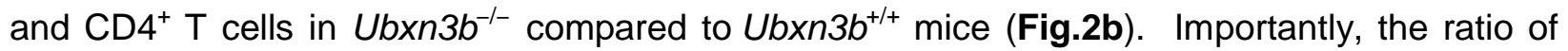
neutrophil-to-T/B lymphocytes $(\mathrm{N} / \mathrm{L})$ in the lung was significantly higher (3.2-fold) in $U b x n 3 b^{-/-}$ (Fig.2c), which is consistent with the clinical observations in severe COVID-19 patients ${ }^{20}$. This prompted us to examine more immune cell compartments in the lung and peripheral blood, and the longer impact of SARS-CoV-2 infection on immune cells. We noted $\sim 3$-fold increase in neutrophil and 10-fold decrease in B cell frequencies (Fig.2d); the N/L ratio was also much higher in the blood of $U b x n 3 b^{-/-}$than $U b x n 3 b^{+/+}$mice at day 3 p.i. (Fig.2e). By day 35 p.i., the total immune cells and T cells were lower, while neutrophils, macrophages/monocytes and N/L ratios were higher, in $U b x n 3 b^{-/-}$than $U b x n 3 b^{+/+}$lungs (Fig.2f, g, Suppl Fig.s2). Remarkably, the B cell frequencies and counts were dramatically decreased (5-20-fold) in both the lung and

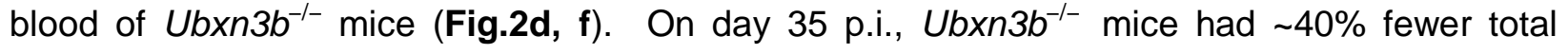
$171 \mathrm{CD}^{+} 5^{+}$cells in the blood (Fig.2h) and presented splenic atrophy characterized by reduced cell 172 density in white pulps and increased myeloid clumps in red pulps, when compared to $U b \times n 3 b^{+/+}$

173 animals (Suppl Fig.s3). Because of the dramatic defect in B cell compartment that might lead to 174 a weak antibody response, we thus measured the concentrations of serum anti- SARS-CoV-2 175 Spike and IVA nucleoprotein IgG by enzyme-linked immunosorbent assay (ELISA). Indeed, the $176 \lg$ concentrations in $U b \times n 3 b^{-/-}$were lower than those in $U b x n 3 b^{+/+}$mice (Fig.2i).

\section{UBXN3B maintains steady-state hematopoietic homeostasis}

179 The above-mentioned results suggest an essential role of UBXN3B in maintenance of immune 180 cell homeostasis during viral infection. Therefore, to test the hypothesis that dysregulated 181 immune homeostasis is caused by $U b \times n 3 b^{-/-}$deficiency, we reasoned that in the steady state 
$182 U b \times n 3 b^{-/-}$mice should have alterations that cannot be explained by infection. Indeed, by flow 183 cytometry, we observed a significant increase in the frequencies of myeloid cells (neutrophils,

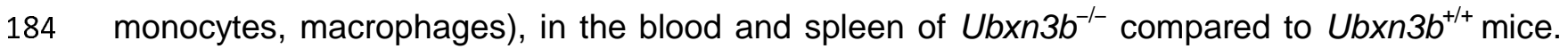
185 Although the T cell frequencies were slightly lower or not different, its counts were significantly

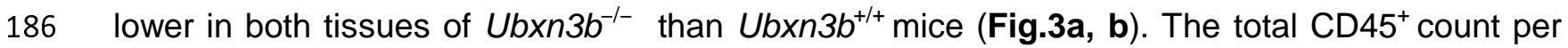
187 spleen was $\sim 3$-fold lower in $U b \times n 3 b^{-/-}$(Fig.3b). Of note, Ubxn3b $b^{-/-}$mice had over 10 times 188 lower B cell counts and frequencies in both tissues of knockout mice (Fig.3a, b). Although the 189 magnitudes of difference in T cell frequency varied with tissues, the $\mathrm{N} / \mathrm{T}$ ratios were uniformly 190 much higher in all tissues of $U b \times n 3 b^{-/-}$when compared to Ubxn3b $b^{+/+}$mice (Fig.3c). Next, we 191 asked if STING has a role in steady state hematopoiesis because our recent study 192 demonstrated a role of UBXN3B in activating STING signaling ${ }^{15}$. The results show that the blood immune cell compositions were similar between wild type and Sting ${ }^{-/-}$mice (Suppl Fig.s4), suggesting a STING-independent role of UBXN3B in hematopoiesis.

Hematopoiesis involves a global change of gene expression controlled by cell-intrinsic 197 transcription factors and epigenetic modifiers, and cell-extrinsic factors such as cytokines, 198 chemokines, growth factors, and interactions with osteoblasts, endothelial cells, reticular cells 199 and stromal cells in its bone marrow niche ${ }^{3}$. To investigate if the B cell defect in Ubxn3b $b^{-/-}$is 200 cell-intrinsic or extrinsic, we performed reciprocal bone marrow transplantation. Firstly, we 201 transferred $\mathrm{Cre}^{+}$Ubxn3b ${ }^{\text {floxflox }}$ bone marrow (CD45.2) to irradiated wild type (WT, CD45.1) 202 recipient mice. Thirty days after transplantation, the recipient WT mice were treated with either 203 corn oil (designated Ubxn3 $b^{+/} \mathrm{BM}-\mathrm{WT}$ ) or tamoxifen (dissolved in corn oil) to induce Ubxn3b 204 deletion in the bone marrow (BM) (designated Ubxn3b-/- BM-WT). We confirmed that $>99 \%$ of 
205

206

207

208

209

210

211

212

213

214

215

216

217

218

219

220

221

222

223

224

225

226

227

blood B cells /neutrophils/monocytes, and $>82 \%$ of T cells were derived from the CD45.2 donor at 45 days after transplantation (Suppl Fig.s5a), indicating successful irradiation and immune reconstitution. We noted an unusually high neutrophil ratio in Ubxn3b $b^{+/+}$BM-WT mice at day 15 than regular $U b \times n 3 b^{+/+}$mice $(\sim 32 \%$ versus $\sim 10 \%)$, and it was back to normal by day 30 (Fig.4a,b, Suppl Fig.s5b). This is likely because of faster reconstitution of neutrophils than B cells after BMT. Nonetheless, the B cell cellularity in the chimeric Ubxn3b $b^{-/-}$BM-WT mice was consistently much lower than that in $U b x n 3 b^{+/+}$BM-WT mice throughout the study period (Fig.4a-c). Moreover, Ubxn3b-/- BM-WT mice were more vulnerable to SARS-CoV-2 infection, typified by more iron-laden cells than $U b x n 3 b^{+/+}$BM-WT mice were in the lung at day 7 p.i (Fig.4d). Conversely, Ubxn3b floxflox or $\mathrm{Cre}^{+} U b x n 3 b^{\text {floxflox }}$ mice (CD45.2) were irradiated and transplanted with WT (CD45.1) bone marrow. Thirty days after transplantation, the recipient mice were treated with tamoxifen, resulting in chimeric WT BM-Ubxn $3 b^{+/+}$and WT BMUbxn3b $b^{-/-}$mice. The B cell numbers were comparable between the two groups (Suppl Fig.s6). These data suggest that UBXN3B plays a cell-intrinsic role in controlling B cell development and hematopoietic UBXN3B is critical for restricting SARS-CoV-2 pathogenesis.

\section{UBXN3B controls B lymphopoiesis by maintaining BLNK protein stability and pre-BCR} signaling

The aforementioned results suggest that UBXN3B likely regulates B cell development. To this end, we quantitated terminally differentiated immune cells in the bone marrow. Among all live cells (after lysis of red blood cells), neutrophil was the most abundant, then B cell. The percentage of B cells was 6-fold lower, while the frequency of neutrophils was moderately higher, in $U b x n 3 b^{-/-}$than $U b x n 3 b^{+/+}$bone marrow (Fig.5a, Suppl Fig.s7a). These results 
suggest that dysregulated hematopoiesis in $U b \times n 3 b^{-/-}$is due to a defect in B lymphopoiesis, which we tested by assessing all the stages of B cell development. Of note, the percentage of

230 large and small precursor Bll (pre-B), immature B (imm-B) and mature B (recirculating B) 231 fractions was significantly lower in $U b x n 3 b^{-/-}$than $U b x n 3 b^{+/+}$, while that of progenitor B (pro-B)

232 and precursor $\mathrm{BI}$ (pre-BI) fractions was the same (Fig.5b, Suppl Fig.s7b), suggesting that 233 UBXN3B is essential for pre-BI transition to pre-Bll, the first checkpoint. Next, we examined 234 stem cells and other lineage progenitors. Total HSCs $\left(\mathrm{Lin}^{-} \mathrm{Sca}^{+} \mathrm{Kit}^{+}\right)$contains two populations, 235 long-term HSCs, which are capable of self-renewal but are quiescent at steady state, and 236 short/mid-term multipotent HSCs (also known as MPPs), which are capable of differentiating 237 into lineage-committed progenitors. The LSK and MPP percentage was modestly decreased in $238 U b \times n 3 b^{-/-}$when compared to $U b x n 3 b^{+/+}$. The frequency of lineage-committed common lymphoid 239 progenitors (CLPs) was also reduced, while the common myeloid progenitors (CMPs) trended 240 higher in Ubxn3b-/- (Fig.5c, Suppl Fig.s8).

242 The abovementioned results demonstrate that UBXN3B is essential specifically for early B cell 243 development, and this is cell-intrinsic. Therefore, we sorted early bone marrow B fractions and 244 quantified by qRT-PCR the mRNA expression of well-established transcription factors for 245 hematopoiesis, several of which are B lineage-specific/dominant transcription factors, including 246 early B cell factor (EBF1), paired box protein 5 (PAX5), myocyte enhancer factor (MEF2C) and 247 Ikaros family zinc finger $1 / 3(\mathrm{IKZF1/3})^{21}$. Ebf1, Pax5 and Ikzf3 (encodes Aiolos) mRNA levels 248 were dramatically induced (>100 fold) in pro-B, pre-B and mature $B$, when compared to those in 249 pre-pro-B cells. Of note, Ikzf3 was decreased by 5-fold and Ikzf1 (encodes lkaros) was modestly 250 reduced but only transiently in $U b \times n 3 b^{-/-}$pro-B cells, when compared to that in $U b \times n 3 b^{+/+}$pro-B 
cells (Suppl Fig.s9a). We also checked B cell surface marker genes (Cd19, Cd79a and II7ra)

252 and observed no significant difference (Suppl Fig.s9b). These data suggest that the

253 transcription factor expression in general is intact in $U b \times n 3 b^{-/-} \mathrm{B}$ lineage.

Because our data have shown that UBXN3B is essential for the transition from pre-BI to large pre-BII (checkpoint 1), we postulated that UBXN3B might regulate pre-B cell receptor (BCR) signaling. Pre-BCR signaling plays several important roles, including allelic exclusion, 258 negative/positive selection, and proliferation of large pre-Bll ${ }^{22}$. A Pre-BCR comprises an Ig $\mu$ 259 heavy chain $(\mu \mathrm{H})$ and a surrogate light chain (SLC); the latter is transiently robustly induced in 260 pro-B and pre-BI, but rapidly down-regulated in large pre-Bll to allow for BCR recombination 261 and expression ${ }^{23}$. Indeed, Vpreb expression (V-set pre-B cell surrogate light chain) was upregulated by $>150$ times in pro-B when compared to pre-pro-B, further in pre-BI cells, then was suppressed in large pre-BII, but barely detected in recirculating B and other fully differentiated 264 lineages. We observed a modest increase in Vpreb mRNA in pro-B, pre-BI and large pre-BII of $265 U b \times n 3 b^{-/-}$, compared to that of Ubxn3b+/+ mice (Fig.6a). However, the surface Vpreb1 protein 266 abundance was comparable between $\mathrm{Vpreb1}^{+}$(pro-B, pre-BI, large pre-BII cells) Ubxn3b $b^{-/-}$and $267 U b x n 3 b^{+/+}$cells (Fig.6b). Of note, Ubxn3b expression was higher in the B lineage than $268 \mathrm{~T} /$ neutrophil/monocytes, being the highest in pre-BI, coincident with its essential role at the first 269 checkpoint (Fig.6a). Next, we attempted to obtain a full picture of the pathways regulated by 270 UBXN3B. To this end, we performed single cell RNA sequencing (scRNAseq) on all bone 271 marrow B fractions (excluding mature, recirculating B), HSCs and progenitors (CLPs, GMPs and 272 MEPs). We analyzed the differentially expressed genes (DEGs) in SLC-high (SLC ${ }^{\text {hi }}$ B subsets 273 (Fig.6c) and identified 49 down-regulated genes with an average count per cell $>1$ and a $p<0.1$ 
274 (Log2 fold range -0.7 to -1.7$)$ in $U b x n 3 b^{-/}$SLC $^{\text {hi }}$ cells. Of note, 10 genes ( 20\% of total down275 regulated DEGs) were related to the cell cycle/mitosis/DNA replication pathways (Suppl Table 276 s1, Fig.6d). The opening of IgK gene locus and recombination by RAG1/2 downstream of pre277 BCR is essential for B lymphopoiesis ${ }^{24,25}$. Intriguingly, Igkc was downregulated too $(\log 2=-1.3$, $278 \mathrm{p}=0.00025)$. Fifty genes were upregulated, including three SLC genes (Vpreb1/2 and Ig/l1)

279 (Suppl Table s1), consistent with the qRT-PCR result (Fig.6a). In Ubxn3b ${ }^{-/}$SLC-low (SLC ${ }^{10}$ ) B 280 cells, 23 genes were significantly down-regulated (Log2 range -0.7 to -2.9 ), three of which were 281 BCR genes (Iglc1, Iglc2, Ighd); but no significant pathway was identified (Suppl Table s2). We 282 noted only 5-7 significantly down-regulated genes and no significantly enriched pathways in 283 HSCs or GMPs (Suppl Table s3, 4). These data suggest that UBXN3B likely regulates pre284 BCR downstream signaling components. To this end, we first examined these protein 285 expression. B-cell linker (BLNK/SLP65) expression was gradually reduced from pre-pro-B to 286 large pre-B cells; it was much lower in $U b \times n 3 b^{-/-}$than that in corresponding $U b \times n 3 b^{+/+} \mathrm{B}$ 287 fractions. The level of Bruton's tyrosine kinase (BTK), phospholipase C gamma 2 (PLC-y2), 288 transcription factors forkhead box protein $\mathrm{O} 1$ (FoxO1) and CCAAT-enhancer-binding protein a $289(\mathrm{C} / \mathrm{EBPa})$ remained constant and similar between all $U b \times n 3 b^{+/+}$and $U b \times n 3 b^{-/-} \mathrm{B}$ fractions 290 (Fig.6e). Blnk mRNA expression was similar between $U b \times n 3 b^{+/+}$and $U b x n 3 b^{-/-}$, but lower in 291 SLC $^{\text {lo }}$ than SLC ${ }^{\text {hi }}$ B cells (Fig.6f). Next we checked if pre-BCR signaling is defective by 292 measuring calcium influx. We purified bone marrow B fractions including SLC ${ }^{\text {hi }}$ pro-B/ pre$293 \mathrm{BI} /$ large pre-BII, SLC ${ }^{10}$ small pre-BII and immature B, and mature B fractions by FACS; 294 stimulated (pre-) BCR signaling with an anti-lgM $\mu \mathrm{H}$ antibody, and monitored calcium flux over a 295 time course by flow cytometry. The anti-lgM $\mu \mathrm{H}$ antibody significantly increased calcium influx in

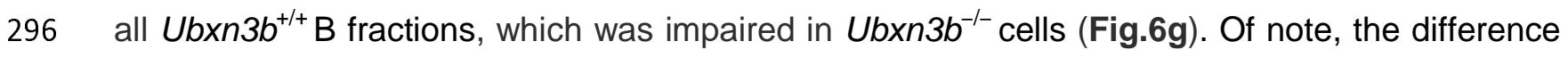




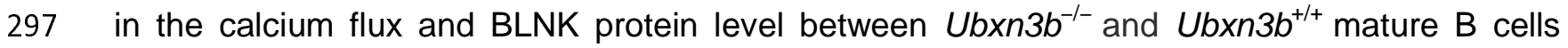

298 became smaller. These data suggest that UBXN3B maintains BLNK protein stability specifically

299 during early B development.

300

\section{DISCUSSION}

302

303 The HSC differentiation cascade must be finely regulated to enable timely production of the right 304 number and type of mature cells, i.e. homeostasis, disruption of which may lead to a 305 pathological state, such as autoimmunity, immunodeficiency, cancer etc. As an example, 306 lymphopenia and a skewing myeloid-to-lymphoid ratio in the elderly may contribute to 307 inflammaging and impaired immunity ${ }^{26}$. In this study, we have discovered a novel and essential 308 role of UBXN3B in maintenance of hematopoietic homeostasis, of note, B cell development, and 309 control of immunopathogenesis of respiratory viral diseases.

The mechanism of UBXN3B action in RNA virus pathogenesis. Mechanistically, UBXN3B

312 might control RNA virus infection by regulating STING signaling and type I IFN responses ${ }^{15} 27$. 313 However, expression of type I IFNs is normal in $U b x n 3 b^{-/-}$mice, so is steady-state 314 hematopoiesis in Sting-deficient mice. These results suggest that the primary function of 315 UBXN3B during RNA virus infection is independent of STING and that dysregulated 316 hematopoiesis may be the main contributor to failure of viral clearance and prolonged 317 immunopathology in $U b \times n 3 b^{-/-}$mice. Indeed, although belonging to very different families of 318 RNA viruses, both SARS-CoV-2 and IVA elicit immunopathology in the lung, including massive 319 immune infiltrates and elevated levels of systemic pro-inflammatory mediators ${ }^{17}$. In particular, 
COVID-19 fatality is strongly associated with neutrophilia and lymphopenia ${ }^{16}$, which is partly

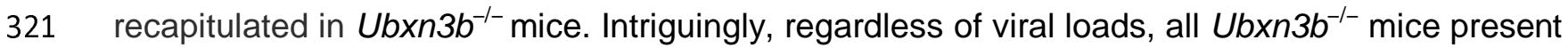

322 a similar degree of hemosiderosis at days 3 and 10 p.i., while none of $U b \times n 3 b^{+/+}$mice have

323 evident hemosiderosis, suggesting that the severe lung damage in $U b \times n 3 b^{-1-}$ mice is primarily

324 caused by immunopathology. On the other hand, heightened immunopathology and tissue

325 damage persists in $U_{b x n 3 b^{-/}}$mice even after viral clearance (day 10 post SARS-CoV-2

326 inoculation), suggesting that immunopathogenesis is disassociated from viral replication.

327 Consistent with this notion, the hyperinflammatory phase of clinical COVID-19 generally

328 happens after the viral load peak, with a few infectious viral particles ${ }^{28}$. At the post clearance

329 stage, a high N/L ratio may sustain inflammation, and thus is correlated with poor prognosis of

330 severe COVID-19 patients ${ }^{29} 20$. The N/L ratio is also the most reliable biomarkers of chronic

331 inflammatory conditions, such as type II diabetes ${ }^{30}$, cardiovascular disease ${ }^{31}$, and aging ${ }^{32}$ etc.

332 These are actually significant risk factors for COVID-19 mortality ${ }^{16}$. These conditions are

333 characterized by a low-grade pro-inflammatory and an "immunosenescence"-like immune state

334 that is unable to clear viruses ${ }^{20}$. In this regard, UBXN3B deficiency might resemble the aging

335 immune state.

337 The mechanism of UBXN3B action in B cell development. Although our initial pursuit with

338 UBXN3B focused on RNA virus pathogenesis, the unexpected and significant phenotype in B 339 cell compartment prompted us to delve into B lymphopoiesis. Mechanistically, this defect is cell

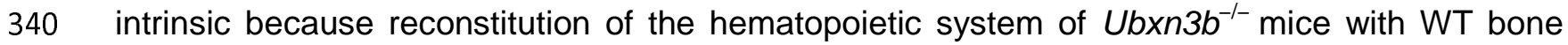
341 marrow restores B cellularity, while reverse transplantation fails to do so. However, expression 342 of cell-intrinsic B-lineage transcription factors (Pax5, Ebf1, Myb, Ikzf1) is largely normal except 
343 for a transient downregulation of Ikzf1/3 in pro-B only, which seems unlikely accountable for a 344 significant defect in the B cell compartment in Ubxn $3 b^{-/-}$mice. Of note, the cellularity of pre-BI 345 and B progenitors remains normal, until the large pre-Bll stage in Ubxn3b-/- mice, suggesting a 346 failure of transition from pre-BI to large pre-BII, also known as the first checkpoint. This stage 347 requires a transient yet essential pre-BCR signaling to drive allelic exclusion, negative/positive 348 selection, and proliferation of large pre-BII ${ }^{22}$. Indeed, in Ubxn $3 b^{-/-}$SLC $^{\text {hi }}$ B subset (primarily 349 pre-BI) ${ }^{33}$, the down-regulated genes are enriched in the cell cycle/mitosis/DNA replication 350 pathways. However, in $U b x n 3 b^{-/-} S^{L} C^{L o} B$ fraction (predominantly small pre-BII and immature B) $351{ }^{33}$, there is no significantly down-regulated pathway. These data suggest that UBXN3B is 352 associated with a developmental stage-specific signaling pathway, e.g., pre-BCR. Indeed, our 353 results demonstrate that UBXN3B is essential for maintenance of BLNK protein stability during 354 the early stage of B development. BLNK is a scaffold protein that is essential for assembly of a 355 macromolecular complex comprising BTK, PLC-y etc. of the (pre-) BCR pathway ${ }^{34}$. BLNK acts 356 at $\mathrm{B}^{2} 20^{+} \mathrm{CD}_{4} 3^{+}$pro-B transition to $\mathrm{B}^{2} 20^{+} \mathrm{CD}^{-} 3^{-}$pre- $\mathrm{B}^{35,36}$. Similarly, with more surface markers, 357 we show that $\mathrm{UBXN3B}$ is essential for $\mathrm{B} 220^{+} \mathrm{CD}_{4} 3^{+}$pre-BI transition to $\mathrm{B}_{220^{+}} \mathrm{CD}_{43^{+}}$large pre$358 \mathrm{BII}$ and then proliferation of $\mathrm{B}^{2} 20^{+} \mathrm{CD}^{-} 3^{-}$small pre-Bll. However, UBXN3B is no longer 359 essential for BLNK protein stability and BCR signaling in mature B cells. This is likely because

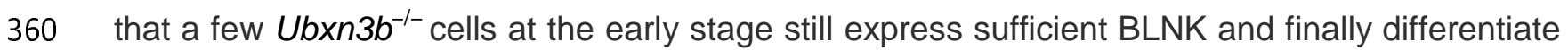
361 into mature B. Considering that UBXN3B is also expressed by mature B cells, it is plausible that 362 UBXN3B may dependent on an early B stage-specific cellular factor to maintain a high BLNK 363 level. 
How does UBXN3B regulate BLNK protein level? Many UBXNs including UBXN3B are known to

366 interact with p97 and multiple E3 ligases, thus controlling newly synthesized protein quality, and

367 regulating protein turnover ${ }^{8,9}$. Thus, by interfacing different E3 ligases under different

368 physiological, developmental or tissue contexts, UBXNs could participate in multiple cellular

369 functions. Indeed, UBXN3B works with tripartite motif-containing 56 (TRIM56) to ubiquitinate

370 and activate STING during DNA virus infection ${ }^{15}$. In early B development, UBXN3B could inhibit

371 a specific E3 ligase that mediates BLNK turnover. In support of this concept, we recently

372 showed that UBXN6 inhibits degradation of phosphorylated tyrosine kinase 2 (TYK2) and type

$373 \mathrm{I} / \mathrm{III}$ interferon receptor activated by type I/III IFNs ${ }^{37}$.

375 In summary, our results demonstrate that UBXN3B is essential for maintenance of 376 hematopoietic homeostasis and in particular B lymphopoiesis during steady state and viral 377 infection. Aberrant immune compartmentalization associated with UBXN3B deficiency may 378 predispose an individual to persistently heightened immunopathology during viral infection. 379 Future work will address how UBXN3B regulates BLNK stability. 


\section{MATERIALS AND METHODS}

\section{Mouse models}

387 The mouse line with the exon 1 of Ubxn3b flanked by two LoxP sites (Ubxn3b ${ }^{\text {floxflox }}$ ) were 388 generated via homologous recombination by Dr. Fujimoto at Nagoya University ${ }^{38}$. The 389 homozygous Ubxn3b $b^{\text {flox/llox }}$ were then crossed with homozygous tamoxifen-inducible Cre 390 recombinase-estrogen receptor T2 mice (The Jackson Laboratory, Stock \# 008463) to generate $391 \mathrm{Cre}^{+} U \mathrm{bxn} 3 b^{\text {floxflox }}$ littermates. To induce Ubxn3b deletion, > 6-weeks old mice were injected 392 with $100 \square \mu l$ of tamoxifen (10 $\square \mathrm{mg} / \square \mathrm{ml}$ in corn oil) (Sigma, \#T5648) via intraperitoneal (i.p.) every 2 days for a total duration of 8 days (4 doses). Successful deletion of Ubxn3b was confirmed in our recent study ${ }^{12}$. A half of $\mathrm{Cre}^{+} \mathrm{Ubxn} 3 b^{\text {flox/flox }}$ litters were treated with tamoxifen and designated $U b \times n 3 b^{-/}$; the other half were treated with corn oil only and designated Ubxn3b+/+ Mice were allowed to purge tamoxifen for at least 4 weeks before any infection or

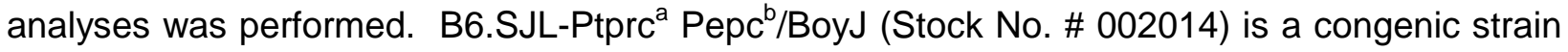
used widely in transplant studies because it carries the differential pan leukocyte marker Ptprc ${ }^{\mathrm{a}}$, commonly known as Cd45.1 or Ly5.1. All experiments were performed in accordance with relevant guidelines and regulations approved by the Institutional Animal Care and Use

401 Committee at the University of Connecticut and Yale University.

404 A rabbit anti-BLNK mAb (Clone D3P2H, Cat \#36438), anti- $\beta$-Actin mAb (Clone D6A8, Cat \# 405 8457), anti-phospho-BLNK mAb (Thr152) (Clone E4P2P, Cat \#62144), anti-GAPDH (Clone 406 D16H11, Cat \# 5174), anti-BTK mAb (Clone D3H5, Cat \# 8547), anti-phospho-BTK mAb 
(Tyr223) (Clone D1D2Z, Cat \# 87457), anti-Syk mAb (Clone D3Z1E, Cat \# 13198), antiphospho-Zap-70 (Tyr319)/Syk (Tyr352) mAb (Clone 65E4, Cat \# 2717), anti-MEK1/2 mAb 409 (Clone D1A5, Cat \# 8727), and anti-phospho-MEK1/2 mAb (Ser221) (Clone 166F8, Cat \# 2338) 410 were purchased from Cell Signaling Technology (Danvers, MA 01923, USA). Human 411 embryonic kidney 293 cells transformed with T antigen of SV40 (HEK293T, \# CRL-3216) and 412 Vero cells (monkey kidney epithelial cells, \# CCL-81) were purchased from American Type 413 Culture Collection (ATCC) (Manassas, VA20110, USA). These cell lines are not listed in the 414 database of commonly misidentified cell lines maintained by ICLAC. Cells were grown in DMEM 415 supplemented with 10\% fetal bovine serum (FBS ) and antibiotics/antimycotics (Life 416 Technologies, Grand Island, NY 14072 USA).We routinely added MycoZAP (Lonza Group, 417 Basel, Switzerland) to cell cultures prevent mycoplasma contamination. SARS-CoV-2 (NR-52281, Isolate USA-WA1/2020) was provided by BEl Resources 419 (funded by National Institute of Allergy and Infectious Diseases and managed by ATCC, United 420 States). The full-length human ACE2 [Accession No: NM_021804.2] cDNA was inserted into 421 pAV-EGFP-CMV/FLAG and Ad5 viruses were prepared by Vector Builder Inc. (Chicago, IL 42260609, USA).

423

424 Concentration of SARS-CoV-2

425 The virus was grown in Vero cells for $72 \mathrm{hrs}$, and the culture medium was cleared by brief 426 centrifugation. A PEG-it Virus Precipitation Solution (Cat\# LV810A-1, System Biosciences, Palo 427 Alto, CA 94303, USA) was added to $40 \mathrm{ml}$ of virus culture at a 1:4 ratio, incubated overnight at $4284^{\circ} \mathrm{C}$. The mixture was centrifuged at $1500 \mathrm{xg}$ for $30 \mathrm{~min}$, and the resulting pellet was suspended 
429 in $1 \mathrm{ml}$ of DMEM medium. In parallel, Vero cell culture medium without virus was processed in

430 the same way and used for mock infection.

432 Plaque-Forming Assay

433 Quantification of infectious viral particles in sera or homogenized tissues was performed on 434 Vero cell monolayer ${ }^{39}$. Briefly, viral samples were incubated with confluent Vero cells (6-well 435 plate) at $37 \square{ }^{\circ} \mathrm{C}$ for $2 \square \mathrm{hr}$. The inoculum was then removed and replaced with $2 \square \mathrm{ml}$ of DMEM 436 complete medium with $1 \%$ SeaPlaque agarose (Cat. \# 50100, Lonza). The cells were incubated 437 at $37 \square{ }^{\circ} \mathrm{C}, 5 \% \mathrm{CO}_{2}$ for 3 days, and on the fourth day the cells were stained with Neutral Red 438 (Sigma-Aldrich) overnight.

\section{Mouse Infection and Monitoring}

441 Mice were administered intranasally $2 \times 10^{8}$ plaque forming units (PFU) of Ad5-hACE2, after 5 442 days then intranasally inoculated with $2 \times 10^{5}$ PFU of SARS-CoV-2 or mock. Three hundred and 443 fifty $\mathrm{CCID}_{50}$ (cell culture infectious dose $50 \%$ assay) of Influenza A PR8/34 H1N1 strain was 444 administered to mice by intranasal instillation in $40 \mu \mathrm{l}$ of sterile phosphate buffered saline. The 445 body mass of individual mice was weighed on the day of infection (Day 0) as a baseline. The 446 percentage change in an animal was calculated as $100 \times$ (Day n-Day 0)/Day 0, where $n$ defines 447 the length of infection (in days).

\section{Bone Marrow Transplantation}

450 Eight weeks or older wild type (WT, B6, Cd45.1, recipient) male mice were irradiated at a lethal 451 dose (900 rad) with a Gammacell-40 irradiator once, and transplanted with ERT2-Cre ${ }^{+}$ 
452 Ubxn3b floxflox bone marrow (BM) cells (donor, Cd4.2) intravenously. Thirty days after

453 transplantation, a half of the mice were administered $100 \mu \mathrm{l}$ of tamoxifen $(10 \square \mathrm{mg} / \square \mathrm{ml}$ in corn oil)

454 (Sigma, \#T5648) via intraperitoneal injection (i.p.) every 2 days for a total duration of 8 days (4 455 doses) (designated Ubxn3b-- BM-WT). The other half was treated with corn oil in the same way 456 (designated Ubxn3 $3 b^{+/+}$BM-WT). Conversely, Ubxn3b flox/flox or ERT2-Cre ${ }^{+}$Ubxn3b floxflox mice 457 were irradiated and transplanted with WT BM. Thirty days after transplantation, all the recipient 458 mice were treated with tamoxifen, resulting in chimeric WT BM-Ubxn3 $3 b^{+/+}$and WT BM-Ubxn3 $3 b^{-/-}$ 459 mice. Fifteen to forty five days after the last dose of tamoxifen, immune cells were analyzed by 460 flow cytometry and/or mice were infected with SARS-CoV-2.

Tissue Histology

463 Tissues were fixed in $4 \%$ paraformaldehyde (PFA), embedded in paraffin, cut into $4 \mu \mathrm{M}$ - thick 464 sections, immobilized to glass slides, decalcified, and processed for hematoxylin and eosin 465 staining. Arbitrary arthritic disease scores (on a 1-5 scale with 1 being the slightest, 5 the worst) 466 were assessed using a combination of histological parameters, including exudation of fibrin and 467 inflammatory cells into the joints, alteration in the thickness of tendons or ligament sheaths, and 468 hypertrophy and hyperlexia of the synovium ${ }^{40}$ in a double-blinded manner. Hemosiderosis was evaluated by iron staining (Prussian Blue stain) (Cat. \# ab150674, 470 from Abcam, Cambridge, CB2 OAX, UK). Lungs were fixed in 4\% PFA, embedded in paraffin, 471 cut into $4 \mu \mathrm{M}$ - thick sections, immobilized to glass slides, deparaffinized in xylene, rinsed with $472100 \%$ ethanol, hydrated progressively in $95 \%, 70 \%$ ethanol and distilled water. The slides were 473 incubated in Iron Stain Solution (1:1 of potassium ferrocyanide solution to hydrochloric acid 474 solution) for $3 \mathrm{~min}$ at ambient temperature, rinsed thoroughly in distilled water, stained in 
475 Nuclear Fast Red Solution for 5 minutes, rinsed again with distilled water 4 times, dehydrated in $47695 \%$ alcohol followed by absolute alcohol, and finally mounted in synthetic resin. The slides 477 were assessed with an Accu-Scope microscope EXI-310 and images were acquired by an 478 Infinity II camera and software.

479

Flow cytometry and Fluorescence-Activated Cell Sorting (FACS)

481 Flow and FACS was performed according to our published study ${ }^{41}$. Mouse tissues were minced 482 with a fine scissor and digested in $4 \mathrm{~mL}$ of digestion medium [20 mg/mL collagenase IV (Sigma483 Aldrich, St. Louis, MO, USA), $5 \mathrm{U} / \mathrm{mL}$ dispase (StemCell, Cambridge, MA, USA), and $50 \mathrm{mg} / \mathrm{mL}$ 484 DNase I mix (Qiagen, Germantown, MD, USA) in complete RPMI1640 medium] at $37^{\circ} \mathrm{C}$ for 4 485 hrs. The lysate was filtrated with a $40 \mu \mathrm{m}$ cell strainer. Cells were then pelleted down by 486 centrifugation at $500 \times \mathrm{g}$ for $5 \mathrm{~min}$. The red blood cells in the cell pellet were lysed three times 487 with a lysis buffer (Cat. \# 420301 from BioLegend, San Diego, CA 92121, USA). Cells were 488 suspended in FACS buffer and stained for 30 min at $4{ }^{\circ} \mathrm{C}$ with the desired antibody cocktails 489 (BioLegend, San Diego, CA, US) of APC-Fire 750-anti CD11b (Cat. \# 101261, clone M1/70), 490 Alexa Fluor 700-anti Ly-6G (Cat. \# 127621, clone 1A8), Brilliant Violet 421-anti CD11c (Cat. \# 491 117343, clone N418), PerCP-Cy5.5-anti MHC II (Cat. \# 107625, clone M5/114.15.2), PE-anti 492 Tetherin (PCDA1) (Cat. \# 12703, clone 10C2), Brilliant Violet 510-anti F4/80 (Cat. \# 123135, 493 clone BM8), APC-anti CD68 (Cat. \# 137007, clone FA-11), PE-Dazzle 594-anti CD3 epsilon 494 (Cat. \# 100347, clone 145-2C11), Brilliant Violet 711-anti CD4 (Cat. \# 100557, clone RM4-5), 495 Brilliant Violet 570-anti CD8a (Cat. \# 100739, clone 53-6.7), Brilliant Violet 650 anti-CD161 496 (NK1.1) (Cat. \# 108735, clone PK136), FITC anti-CD117 (cKit) (Cat. \# 105805, clone 2B8), PE 497 anti- erythroid cells (Cat. \# 116207, clone TER-119), Brilliant Violet 711-anti CD115 (Cat. \# 
135515, clone AFS98), FITC-anti CD25 (Cat. \# 102005, clone PC61), Zombie UV (Cat. \# 423107), PE-Cy7-anti CD45 (Cat. \# 103113, clone 30-F11), TruStain FcX-anti CD16/32 (Cat. \# 500 101319, clone 93), APC anti-CD127 (IL-7Ra) (Cat. \# 135011, clone A7R34), PE-Dazzle 594 501 anti-Sca-1 (Ly-6A/6E) (Cat. \# 108137, clone D7), PE-Cy5 anti-Flt-3 (CD135) (Cat. \# 135311, 502 clone A2F10), Brilliant Violet 421-anti CD34 (Cat. \# 119321, clone MEC14.7), PE anti-CD16/32 503 (Cat. \# 101307, clone 93), Brilliant Violet 711-anti IgM (Cat. \# 406539, clone RMM-1), Brilliant 504 Violet 421-anti CD45R (B220) (Cat. \# 103239, clone RA3-6B2), Alexa Fluor 700-anti CD19 (Cat. 505 \# 115527, clone 6D5), PE-Cy7 anti-CD93 (Cat. \# 136505, clone AA4.1) and Lin- (anti-CD4, CD8, 506 CD11b, CD11c, Gr1, NK1.1, TER119, Singlec-F, FceRla, CD19, B220 cocktail). After staining 507 and washing, the cells were fixed with 4\% PFA and analyzed on a Becton-Dickinson FACS 508 ARIA II, CyAn advanced digital processor (ADP). Data were analyzed using the FlowJo 509 software. Among CD45 cells, CD11 $\mathrm{b}^{+}$Ly6G $^{+}$cells were classified as neutrophils, Ly6G CD11 b

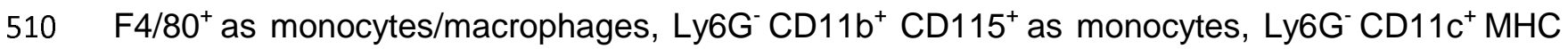
$511 \mathrm{II}^{+}$as dendritic cells (DC), $\mathrm{CD}^{+}$as total T cells, $\mathrm{CD} 3^{+} \mathrm{CD} 4^{+}$as $\mathrm{CD} 4 \mathrm{~T}$ cells, $\mathrm{CD} 3^{+} \mathrm{CD} 8^{+}$as $\mathrm{CD} 8$ $512 \mathrm{~T}$ cells, $\mathrm{CD}_{19}{ }^{+}$as B cells. $\mathrm{Lin}^{-} \mathrm{Sca}^{+} \mathrm{cKit}^{+}$cells were identified as total HSCs, which were 513 subdivided into Flt3 ${ }^{\text {high }}$ (short-term HSC or MPP) and Flt3 ${ }^{\text {low }}$ (long-term HSC). Lin Sca $^{-}$cKit $^{+}$cells 514 were subdivided into $\mathrm{CD} 34^{\text {low }} \mathrm{CD} 16 / 32^{\text {low }}(\mathrm{MEP}), \mathrm{CD} 34^{\text {high }} \mathrm{CD} 16 / 32^{\text {low }}(\mathrm{CMP})$ and $\mathrm{CD} 34^{\text {low }}$ 515 CD16/32 $2^{\text {high }}\left(\right.$ GMP). The Lin $^{-} \mathrm{CD}_{12} 7^{+} \mathrm{CKit}^{+}$cells were identified as CLP.

516 To analyze B lineage fractions, non-B cells (after lysis of red blood cells) were first 517 dumped with FITC-CD3, -TER119, -LY6G, -LY6C, -CD11b, and -NK1.1. The remaining cells 518 were then sequentially gated on BV650 anti-B220 (Cat. \# 103241, clone RA3-6B2), APC anti519 CD43 (Cat. \# 143207, clone S11), PerCP-Cy5 anti-CD24 (Cat. \# 101824, clone M1/69), PE anti520 BP1/CD249/Ly51 (Cat. \# 108307, clone 6C3), BV421 anti-lgM (Cat. \# 406517, clone RMM-1), 
521

522

523

524

525

526

527

528

529

530

531

532

533

534

535

536

537

538

539

540

541

542

543

APC-Cy7 anti-lgD (Cat. \# 405715, clone 11-26c.2a), PE-Cy7 anti-CD93 (Cat. \# 136505, clone RAA4.1), PE-Dazzle 594 anti-CD19 (Cat. \# 115553, clone 6D5).

\section{Single cell RNA sequencing (scRNA-seq)}

Bone marrows were pooled from three mice/genotype, and red blood cells were lysed. HSCs/progenitors and B subsets (excluding mature B) were sorted as described above. In order to obtain an even coverage of each cell compartment/subset, we mixed approximately $1 / 6$ of pre- $B, 1 / 4$ of immature $B$ (which are much more abundant than the others are) with all the HSCs/progenitors (including B progenitors). About $5 \times 10^{4}$ live cells were subjected to a dropletbased 10x Genomics chromium single cell RNA-Seq on a NovaSeq 6000 sequencer, and

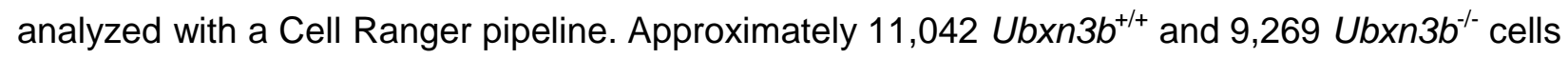
were sequenced and; 1600 gene/cell and 3800 unique molecular identifier (UMI) counts/cell were obtained. Clustering cells, annotating cell clusters and analyzing differentially expressed genes were performed with Loupe Browser 5.0.1. For annotating cell clusters, we did not merely rely on the surface markers for flow cytometry, rather we referred to a database, Bloodspot, which provides transcript expression profiles of genes and gene signatures in healthy and malignant hematopoiesis and includes data from both humans and mice ${ }^{42}$. The $B$ subsets were readily identified by a medium to high level of B-restricted transcription factors (Pax5, Ebf1) and surface markers (Cd19, Cd79a), while a barely detectable level of Fcgr3 and

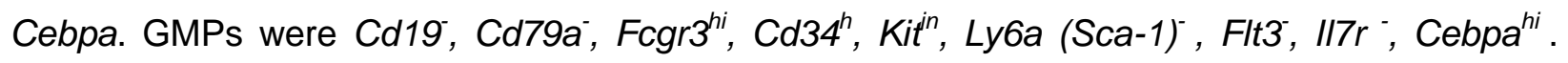

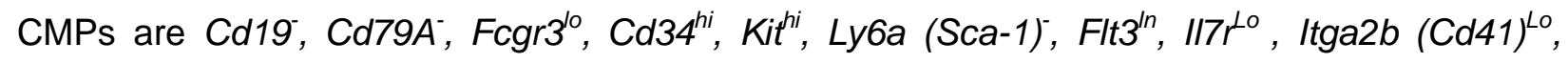

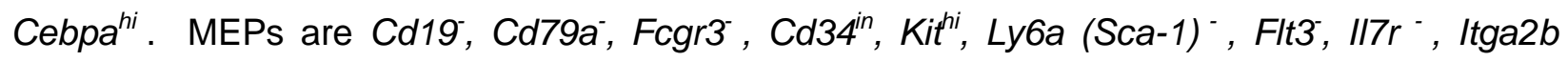

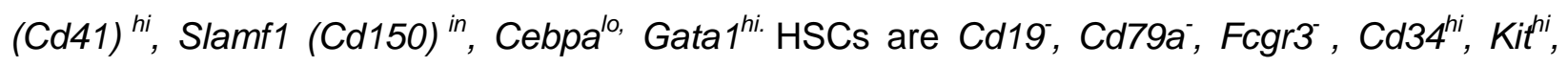


544 Ly6a $(\mathrm{Sca}-1){ }^{\text {in }}$, Flt3 ${ }^{\text {in }}, \mathrm{II7r}$ : Bioinformatics analyses were performed using Reactome 545 (https://reactome.org/). Only those genes with an average count of greater than one per cell and 546 a $p<0.1$ were analyzed.

Calcium Flux Assay

549 Bone marrow B factions were sorted and calcium influx was assayed with a Fluo-4 Direct ${ }^{\mathrm{TM}}$ 550 Calcium Assay Kit (ThermoFisher cat\# F10471). Briefly cells were incubated with Fluo-4 551 Direct $^{\mathrm{TM}}$ at $37^{\circ} \mathrm{C}$ for 1 hour. The cells were stimulated with $20 \mu \mathrm{g} / \mathrm{ml}$ of a purified $\mathrm{F}\left(\mathrm{abb}^{\prime}\right) 2$ goat 552 anti-mouse $\operatorname{lgM}\left(\mu\right.$ chain) antibody (BioLegend, Clone Poly21571, Cat\# 157102) at $37^{\circ} \mathrm{C}$ for 10 553 seconds, and were immediately analyzed by flow cytometry on a Becton-Dickinson FACS ARIA 554 II, CyAn advanced digital processor (ADP). The final results were presented as the ratio of the 555 mean fluorescence intensity at any given time point $[F]$ subtracted by the fluorescence at time 556 point zero [F0] (before stimulation) and divided by F0, i.e., $\Delta F / F 0$.

557

559 We used a LEGENDPlex (BioLegend, San Diego, CA 92121, USA) bead-based immunoassay 560 to quantify the cytokine concentrations in the sera of SARS-CoV-2 infected mice. The 561 procedures were exactly same as described in the product manual. Briefly, the samples were 562 mixed with antibody-coated microbeads in a filter-bottom microplate, and incubated at room 563 temperature for $2 \mathrm{hrs}$ with vigorous shaking at $500 \mathrm{rpm}$. After removal of unbound analytes and 564 two washes, $25 \mu \mathrm{L}$ of detection antibody was added to each well, and the plate was incubated 565 at room temperature for $1 \mathrm{hr}$ with vigorous shaking at $500 \mathrm{rpm}$. Twenty-five $\mu \mathrm{L}$ of SA-PE reagent 566 was then added directly to each well, and the plate was incubated at room temperature for 
$56730 \mathrm{~min}$ with vigorous shaking at $500 \mathrm{rpm}$. The beads were washed twice with wash buffer, and 568 then transferred to a microfuge tube. The beads were fixed with $4 \%$ PFA for $15 \mathrm{~min}$ and 569 resuspended in an assay buffer. The beads were run through a BIORAD ZE5 and the 570 concentrations of analytes were calculated with the standards included using a LEGENDPlex 571 software.

572

573 Quantification of serum IgG by Enzyme-Linked ImmunoSorbent Assay (ELISA)

574 Anti-SARS-CoV-2 Spike IgG titers were measured with a commercial ELISA kit (Acro 575 Biosystems, Cat \# RAS-T018). For quantification of influenza lgG, one nanogram of 576 recombinant A/PR/8/34 influenza NP (generated by UConn Health Protein Expression Core) in $577100 \mu \mathrm{L}$ of coating buffer (0.05 M carbonate-bicarbonate, $\mathrm{pH} 9.6)$ was coated to a 96 -well 578 microplate at $4^{\circ} \mathrm{C}$ overnight. The plate was washed once with a wash solution (50 mM Tris, 0.14 $579 \mathrm{M} \mathrm{NaCl}, 0.05 \%$ Tween 20, $\mathrm{pH} 8.0$ ), and blocked with $4 \%$ bovine serum albumin at room 580 temperature for $2 \mathrm{hrs}$. $100 \mu \mathrm{L}$ of each diluted serum specimens (500-fold) was added a well and 581 incubated at room temperature for $1 \mathrm{hr}$, then the unbound serum was washed off three times 582 with the wash solution. $100 \mu \mathrm{L}$ of diluted horseradish peroxidase-conjugated goat anti-mouse 583 lgG was added to each well and incubated at room temperature for $1 \mathrm{hr}$. After stringency wash, $584100 \mu \mathrm{L}$ of substrate 3,3',5,5' - tetramethylbenzidine (TMB) was added to each well and 585 incubated at room temperature for 5-30min for color development, and terminated by $100 \mu \mathrm{L}$ of $5860.16 \mathrm{M}$ sulfuric acid. The absorption at wavelength $450 \mathrm{~nm}\left(\mathrm{~A}_{450 \mathrm{~nm}}\right)$ was read on a Cytation 1 587 plate reader (BioTek, Winooski, VT, USA).

588

589 Immunoblotting 
590 To prepare B cell fractions for immunoblotting, bone marrow B cells were sorted by flow 591 cytometry as described above $\left(\sim 10^{4}-10^{5}\right.$ cells each fraction), pelleted down by brief 592 centrifugation, suspended in $50 \mu \mathrm{L}$ of $2 x$ SDS-PAGE sample buffer, boiled at $95^{\circ} \mathrm{C}$ for $5 \mathrm{~min}$, and 593 centrifuged at $13,000 x$ for $10 \mathrm{~min}$. Immunoblotting was performed using standard procedures. 594 Briefly, protein samples were resolved by SDS-PAGE (sodium dodecyl sulfate-polyacrylamide 595 gel electrophoresis, $4-20 \%$ gradient) and transferred to a nitrocellulose membrane. The 596 membrane was blocked in 5\% fat-free milk at room temperature for one hour, incubated with a 597 primary antibody over night at $4^{\circ} \mathrm{C}$, washed briefly and incubated with an HRP-conjugated 598 secondary antibody for 1 hour at room temperature. An ultra-sensitive or regular enhanced 599 chemiluminescence (ECL) substrate was used for detection (ThermoFisher, Cat\# 34095, 32106). 600 For immunoblotting of proteins from an extremely low number of sorted bone marrow B cells, a 601 Lumigen ECL substrate ought to be used (Southfield, Michigan 48033, USA).

602

Reverse Transcription and Quantitative (q) PCR

604 Up to $1 \square \times \square 10^{6}$ cells or $10 \mathrm{mg}$, tissues were collected in $350 \square \mu$ of RLT buffer (QIAGEN RNeasy 605 Mini Kit). RNA was extracted following the QIAGEN RNeasy manufacturer's instructions. 606 Reverse transcription of RNA into complementary DNA (cDNA) was performed using the BIO607 RAD iScript ${ }^{\mathrm{TM}}$ cDNA Synthesis Kit. Quantitative PCR (qPCR) was performed with gene-specific 608 primers and SYBR Green PCR master mix. Results were calculated using the $-\Delta \Delta \mathrm{Ct}$ method 609 and a housekeeping gene, beta actin, as an internal control. The qPCR primers and probes for 610 immune genes were reported in our previous studies ${ }^{15,27,43}$. The new primers are listed in 611 Table 1.

612 


\section{Data Acquisition and Statistical Analysis}

614 The sample size chosen for our animal experiments in this study was estimated according to

615 our prior experience in similar sets of experiments and power analysis calculations

616 (http://isogenic.info/html/power_analysis.html). All animal results were included and no method

617 of randomization was applied. All data were analyzed with a GraphPad Prism software by non-

618 parametric Mann-Whitney test or two-tailed Student's $t$-test depending on the data distribution.

619 The survival curves were analyzed by a Log-Rank test. $P$ values of $\leq 0.05$ were considered

620 statistically significant.

621 


\section{Footnotes}

623

624 Author contribution: T.G designed and performed the majority of the experimental procedures 625 and data analyses. D.Y. helped T.G. with most of the experimental procedures. T.L. and A.G.H. 626 contributed to some of the experimental procedures. B.W. contributed to flow cytometry 627 analysis. B.T. and L.H. helped to acquire the influenza data. K.W provided guidance to bone 628 marrow transplantation experiments. Y.W., L.Y., G.C., L.H., A.T.V. and E.F. contributed to 629 discussion, data interpretations and/or helped to improve writing. P.W. conceived and oversaw 630 the study. T.G. and P.W. wrote the paper and all the authors reviewed and/or modified the 631 manuscript.

632

633 Funding Source: This project was funded in part by National Institutes of Health grants to P. W, 634 R01Al132526 and R21Al155820, and an UConn Health startup fund to P.W.

636 Conflict of Interest: No financial or non-financial interest to disclose.

637

638 Data availability: All data generated or analysed during this study are included in this published 639 article (and its supplementary information files). The raw RNAseq data is available at 640 https://www.ncbi.nlm.nih.gov/geo/, GEO \# (pending)

642 Biological materials: All unique materials used are readily available from the authors. However, 643 the availability of live animals may change over time. 
bioRxiv preprint doi: https://doi.org/10.1101/2021.03.04.433919; this version posted August 24, 2021. The copyright holder for this preprint (which was not certified by peer review) is the author/funder. All rights reserved. No reuse allowed without permission.

645

646 


\begin{tabular}{|c|c|c|}
\hline $\begin{array}{l}647 \\
648\end{array}$ & \multicolumn{2}{|c|}{ References } \\
\hline 649 & 1 & Boettcher, S. \& Manz, M. G. Regulation of Inflammation- and Infection-Driven \\
\hline 650 & & Hematopoiesis. Trends Immunol 38, 345-357, doi:10.1016/j.it.2017.01.004 (2017). \\
\hline 51 & 2 & Dancey, J. T., Deubelbeiss, K. A., Harker, L. A. \& Finch, C. A. Neutrophil kinetics in man. \\
\hline 652 & & J Clin Invest 58, 705-715, doi:10.1172/JCl108517 (1976). \\
\hline $\begin{array}{l}653 \\
654\end{array}$ & 3 & $\begin{array}{l}\text { Rieger, M. A. \& Schroeder, T. Hematopoiesis. Cold Spring Harb Perspect Biol 4, } \\
\text { doi:10.1101/cshperspect.a008250 (2012). }\end{array}$ \\
\hline $\begin{array}{l}655 \\
656\end{array}$ & 4 & $\begin{array}{l}\text { Busch, K. et al. Fundamental properties of unperturbed haematopoiesis from stem cells } \\
\text { in vivo. Nature } 518,542-546 \text {, doi: } 10.1038 / \text { nature } 14242(2015) \text {. }\end{array}$ \\
\hline 657 & 5 & $\begin{array}{l}\text { Rodriguez-Fraticelli, A. E. et al. Clonal analysis of lineage fate in native haematopoiesis. } \\
\text { Nature } 553,212-216 \text {, doi: } 10.1038 / \text { nature } 25168 \text { (2018). }\end{array}$ \\
\hline $\begin{array}{l}659 \\
660\end{array}$ & 6 & $\begin{array}{l}\text { Melchers, F. Checkpoints that control B cell development. J Clin Invest 125, 2203-2210, } \\
\text { doi:10.1172/JCI78083 (2015). }\end{array}$ \\
\hline 661 & 7 & Schuberth, C. \& Buchberger, A. UBX domain proteins: major regulators of the AAA \\
\hline $\begin{array}{l}662 \\
663\end{array}$ & & $\begin{array}{l}\text { ATPase Cdc48/p97. Cell Mol Life Sci 65, 2360-2371, doi:10.1007/s00018-008-8072-8 } \\
\text { (2008). }\end{array}$ \\
\hline 664 & 8 & $\begin{array}{l}\text { Kondo, H. et al. p47 is a cofactor for p97-mediated membrane fusion. Nature } 388,75-78 \\
\text { (1997). }\end{array}$ \\
\hline 666 & 9 & Alexandru, G. et al. UBXD7 binds multiple ubiquitin ligases and implicates p97 in \\
\hline 667 & & HIF1alpha turnover. Cell 134, 804-816, doi:10.1016/j.cell.2008.06.048 (2C \\
\hline $\begin{array}{l}668 \\
669 \\
670\end{array}$ & 10 & $\begin{array}{l}\text { Wang, P. et al. UBXN1 interferes with Rig-l-like receptor-mediated antiviral immune } \\
\text { response by targeting MAVS. Cell Rep 3, 1057-1070, doi:10.1016/j.celrep.2013.02.027 } \\
\text { (2013). }\end{array}$ \\
\hline 671 & 11 & Kim, J. H. et al. FAS-associated factor-1 positively regulates type I interferon response \\
\hline 672 & & to RNA virus infection by targeting NLRX1. PLoS Path \\
\hline 673 & & doi:10.1371/journal.ppat.1006398 (2017). \\
\hline 674 & 12 & Dai, T. et al. FAF1 Regulates Antiviral Immunity by Inhibiting MAVS but Is Ant \\
\hline $\begin{array}{l}675 \\
676\end{array}$ & & $\begin{array}{l}\text { by Phosphorylation upon Viral Infection. Cell Host Microbe 24, 776-790 e775, } \\
\text { doi:10.1016/i.chom.2018.10.006 (2018). }\end{array}$ \\
\hline 677 & 13 & Wang, Y. B. et al. Ubiquitin-associated Domain-containing UBX Protein UBXN1 is a \\
\hline 678 & & Negative Regulator of NF-kappaB Signaling. J Biol Chem, doi:10.1074/jbc.M114.631689 \\
\hline 679 & & \\
\hline 680 & 14 & et a \\
\hline 681 & & \\
\hline 682 & & e1006187, doi:10.1371/journal.ppat.1006187 (2017). \\
\hline 683 & 15 & Yang, L. et al. UBXN3B positively regulates STING-mediated antiviral i \\
\hline 684 & & 1467-018-04759-8 (2018). \\
\hline 685 & 16 & Harrison, A. G., Lin, T. \& Wang, P. Mechanisms of SARS-CoV-2 Transmission and \\
\hline 686 & & Pathogenesis. Trends Immunol 41, 1100-1115, doi:10.1016/j.it.2020.10.004 (2020). \\
\hline 687 & 17 & Bai, Y. \& Tao, X. Comparison of COVID-19 and influenza characteristics. J Zhejiang \\
\hline 688 & & Univ Sci B 22, 87-98, doi:10.1631/jzus.B2000479 (2021). \\
\hline 689 & 18 & Sun, J. et al. Generation of a Broadly Useful Model for COVID- \\
\hline 69 & & nation, and Treatment. Cell 182, 734-743 e735, doi:10.1016/j.cell.2020.C \\
\hline 60 & & \\
\hline
\end{tabular}


729

730

731

732

733

734

735

736

737

19 Hassan, A. O. et al. A SARS-CoV-2 Infection Model in Mice Demonstrates Protection by Neutralizing Antibodies. Cell 182, 744-753 e744, doi:10.1016/j.cell.2020.06.011 (2020).

20 de Candia, P., Prattichizzo, F., Garavelli, S. \& Matarese, G. T Cells: Warriors of SARSCoV-2 Infection. Trends Immunol 42, 18-30, doi:10.1016/j.it.2020.11.002 (2021).

21 Choukrallah, M. A. \& Matthias, P. The Interplay between Chromatin and Transcription Factor Networks during B Cell Development: Who Pulls the Trigger First? Front Immunol 5, 156, doi:10.3389/fimmu.2014.00156 (2014).

22 Martensson, I. L., Almqvist, N., Grimsholm, O. \& Bernardi, A. I. The pre-B cell receptor checkpoint. FEBS Lett 584, 2572-2579, doi:10.1016/j.febslet.2010.04.057 (2010).

23 Dul, J. L. et al. The murine VpreB1 and VpreB2 genes both encode a protein of the surrogate light chain and are co-expressed during B cell development. Eur J Immunol 26, 906-913, doi:10.1002/eji.1830260428 (1996).

24 Mandal, M. et al. Ras orchestrates exit from the cell cycle and light-chain recombination during early B cell development. Nat Immunol 10, 1110-1117, doi:10.1038/ni.1785 (2009).

25 Shaw, A. C., Swat, W., Davidson, L. \& Alt, F. W. Induction of lg light chain gene rearrangement in heavy chain-deficient B cells by activated Ras. Proc Natl Acad Sci U S A 96, 2239-2243, doi:10.1073/pnas.96.5.2239 (1999).

26 Groarke, E. M. \& Young, N. S. Aging and Hematopoiesis. Clin Geriatr Med 35, 285-293, doi:10.1016/j.cger.2019.03.001 (2019).

27 Geng, T. et al. A critical role for STING signaling in limiting pathogenesis of Chikungunya virus. J Infect Dis, doi:10.1093/infdis/jiaa694 (2020).

$28 \mathrm{He}, \mathrm{X}$. et al. Temporal dynamics in viral shedding and transmissibility of COVID-19. Nat Med 26, 672-675, doi:10.1038/s41591-020-0869-5 (2020).

29 Liu, J. et al. Neutrophil-to-lymphocyte ratio predicts critical illness patients with 2019 coronavirus disease in the early stage. J Trans/ Med 18, 206, doi:10.1186/s12967-02002374-0 (2020).

30 Guo, X. et al. Neutrophil:lymphocyte ratio is positively related to type 2 diabetes in a large-scale adult population: a Tianjin Chronic Low-Grade Systemic Inflammation and Health cohort study. Eur J Endocrinol 173, 217-225, doi:10.1530/EJE-15-0176 (2015).

31 Angkananard, T., Anothaisintawee, T., McEvoy, M., Attia, J. \& Thakkinstian, A. Neutrophil Lymphocyte Ratio and Cardiovascular Disease Risk: A Systematic Review and Meta-Analysis. Biomed Res Int 2018, 2703518, doi:10.1155/2018/2703518 (2018).

$32 \mathrm{Li}, \mathrm{J}$. et al. Neutrophil-to-Lymphocyte Ratio Positively Correlates to Age in Healthy Population. J Clin Lab Anal 29, 437-443, doi:10.1002/jcla.21791 (2015).

33 Martensson, I. L. \& Ceredig, R. Review article: role of the surrogate light chain and the pre-B-cell receptor in mouse B-cell development. Immunology 101, 435-441, doi:10.1046/j.1365-2567.2000.00151.x (2000).

34 Hendriks, R. W. \& Middendorp, S. The pre-BCR checkpoint as a cell-autonomous proliferation switch. Trends Immunol 25, 249-256, doi:10.1016/j.it.2004.02.011 (2004).

35 Minegishi, Y. et al. An essential role for BLNK in human B cell development. Science 286, 1954-1957, doi:10.1126/science.286.5446.1954 (1999).

36 Pappu, R. et al. Requirement for B cell linker protein (BLNK) in B cell development. Science 286, 1949-1954, doi:10.1126/science.286.5446.1949 (1999).

37 Ketkar, H. et al. UBX Domain Protein 6 Positively Regulates JAK-STAT1/2 Signaling. J Immunol 206, 2682-2691, doi:10.4049/jimmunol.1901337 (2021). 
38 Imai, N. et al. Hepatocyte-Specific Depletion of UBXD8 Induces Periportal Steatosis in Mice Fed a High-Fat Diet. PLoS One 10, e0127114, doi:10.1371/journal.pone.0127114 (2015).

39 Wang, P. et al. Caspase-12 controls West Nile virus infection via the viral RNA receptor
RIG-I. Nat Immunol 11, 912-919, doi:10.1038/ni.1933 (2010).

40 Chen, N. et al. Epidemiological and clinical characteristics of 99 cases of 2019 novel coronavirus pneumonia in Wuhan, China: a descriptive study. The Lancet 395, 507-513, doi:https://doi.org/10.1016/S0140-6736(20)30211-7 (2020).

41 Lin, T. et al. CXCL10 Signaling Contributes to the Pathogenesis of Arthritogenic Alphaviruses. Viruses 12, doi:10.3390/v12111252 (2020).

42 Bagger, F. O. et al. BloodSpot: a database of gene expression profiles and transcriptional programs for healthy and malignant haematopoiesis. Nucleic Acids Res 44, D917-924, doi:10.1093/nar/gkv1101 (2016).

43 Yang, L. et al. Macrophage scavenger receptor 1 controls Chikungunya virus infection through autophagy in mice. Commun Bio/ 3, 556, doi:10.1038/s42003-020-01285-6

753 (2020).

754

755 


\section{FIGURE LEGEND}

\section{Fig.1 UBXN3B is essential for controlling SARS-CoV-2 and influenza pathogenesis. a-d)} Sex-and-age matched mice were administered $2 \times 10^{5}$ plaque forming units (PFU) of SARS-CoV-

2 intranasally. a) Percentage changes in the body mass of mock-treated $\mathrm{Cre}^{+}$Ubxn3b $\mathrm{b}^{\text {floxflox }}$

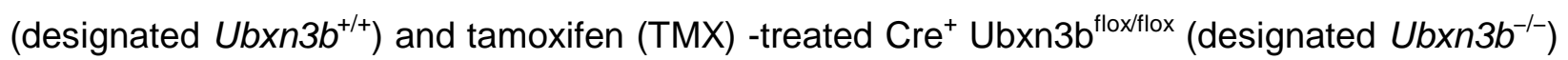
littermates, during the course of SARS-CoV-2 infection. Data point: mean \pm s.e.m, $N=6-8$. *, $\mathrm{p}<0.05 ;{ }^{* *}, \mathrm{p}<0.01 ;{ }^{* * *}, \mathrm{p}<0.001$ (two-tailed Student's $t$-test). b) Representative micrographs of hematoxylin and eosin staining (H\&E) of lung sections from mock or SARS-CoV-2 infected mice on day 3 and 10 post infection (p.i.). The green arrow points to a cluster of immune infiltrates. The red arrow indicates a cluster of brownish cells of hemosiderosis. Magnification $400 \mathrm{x}$. c) Iron-staining (blue) of lung sections from mock or SARS-CoV-2 infected mice on days 3 and 10p.i. Black arrows point to iron laden cells. Mock: mock infected. $\mathrm{N}=2$ (mock), 4 (Day 3), 7 (Day 10), 3 (Day 35) per genotype. e, f) Sex-and-age matched mice were administered $350 \mathrm{CCID}_{50}$ (cell culture infectious dose 50\% assay) influenza A PR/8/34 H1N1 intranasally. e) The percentage of the body mass relative to day 0 (weighed immediately before infection). Data point: mean \pm s.e.m. $N=5-6$. f) The survival curve. $N=6$ per genotype. $P=0.02$ (Log-Rank test).

\section{Fig.2 UBXN3B is essential for immune cell homeostasis during SARS-CoV-2 infection.}

Sex-and-age matched mice were administered $2 \times 10^{5}$ plaque forming units (PFU) of SARS-CoV2 intranasally. a) Total $\mathrm{CD}_{4} 5^{+}$cells, b) the percentage (relative to $\mathrm{CD} 45^{+}$cells) of various immune cell populations quantified by flow cytometry, c) the neutrophil-to-T cell ratio, in one lung of SARS-CoV-2 infected mice at day 3 post infection (p.i.). d) The percentage (relative to 
780

781

782

783

784

785

786

787

788

789

790

791

792

793

794

795

796

797

798

799

800

801

802

CD $45^{+}$cells) of various immune cell compartments, and $\left.\mathbf{e}\right)$ the neutrophil-to-B/T cell ratios (N/B, $\mathrm{N} / \mathrm{T}$ ), in the blood at day 3 post infection (p.i.). The cell counts and percentage of various immune cell populations $\mathbf{f}$ ) and $\mathbf{g}$ ) the neutrophil-to-B/T cell ratios in one lung, $\mathbf{h}$ ) cell counts in the blood at day 35 p.i. i) The concentrations of serum IgG against SARS-CoV-2 Spike and influenza A PR/8/34 H1N1 NP were quantitated by ELISA and presented as optical density at 450nm $\left(O . D_{450 \mathrm{~nm}}\right)$. Neu: neutrophil, Mac/Mono: macrophage/monocyte, DC: dendritic cell. Each symbol=one mouse. ${ }^{*}, \mathrm{p}<0.05 ;{ }^{* *}, \mathrm{p}<0.01 ;{ }^{* * *}, \mathrm{p}<0.001$ (non-parametric Mann-Whitney test). The horizontal line indicates the median of the result.

Fig.3 UBXN3B is essential for steady-state immune cell homeostasis. The percentage (relative to $\mathrm{CD} 45^{+}$cells) of various immune cell populations and cell counts were quantified by flow cytometry in the a) blood and b) spleen of specific pathogen-free littermates. c) The neutrophil to B/T cell ratios. Neu: neutrophil, Mac/Mono: macrophage/monocyte, DC: dendritic cell, Mast: mast cell, Eos: eosinophil, NK: natural killer, NKT: natural killer T cells. Each symbol=one mouse. The horizontal line indicates the median of the result. ${ }^{*}, p<0.05 ;{ }^{* *}, p<0.01$; ${ }^{* * *}, \mathrm{p}<0.001$ (two-tailed Student's $t$-test).

Fig.4 The essential role of UBXN3B for B cell development is cell-intrinsic. Irradiated wild type (WT, CD45.1) recipient mice were transplanted with $\mathrm{Cre}^{+} U b x n 3 b^{\mathrm{f} / \mathrm{f}}$ bone marrow (CD45.2). The mice were then treated with tamoxifen (TMX) to delete Ubxn3b in hematopoietic cells

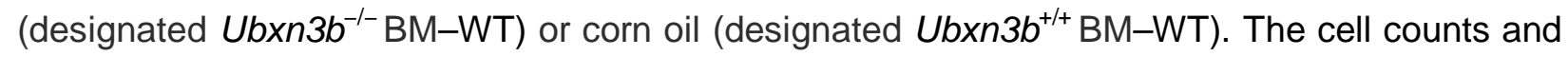
percentage (relative to $\mathrm{CD}^{+} 5^{+}$cells) of various immune cell populations in the blood were quantified by flow cytometry at days a) 15 , b) 30 , c) 45 after completion of the TMX treatment. d) 
803

804

805

806

807

808

809

810

811

812

813

814

815

816

817

818

819

820

821

822

823

824

825

Iron-staining (blue) of lung sections at day 7 p.i. The black arrow points to iron-laden cells. Magnification: 100x. $\mathrm{N}=5$. Each symbol=one mouse. The horizontal line indicates the median of the result. ${ }^{*}, \mathrm{p}<0.05 ;{ }^{* *}, \mathrm{p}<0.01 ;{ }^{* *}, \mathrm{p}<0.001$ (two-tailed Student's $t$-test).

Fig.5 UBXN3B is essential for pre-BI transition to pre-Bll. The frequencies of a) terminally differentiated immune cells and $\mathbf{b}$ ) stem cells/progenitors, quantified by flow cytometry (relative to live cells after lysis of red blood cells). c) The frequencies and cellularity of B lineage subsets in the bone marrow of specific pathogen-free littermates. Neu: neutrophil, Mono: monocyte, DC: dendritic cell, NK: natural killer, LSK: Lin ${ }^{-} \mathrm{Sca}^{+} \mathrm{Kit}^{+}$, LT-HSC: long-term hematopoietic stem cell, ST-HSC: short-term multipotent HSC (also known as MPP), CMP: common myeloid progenitor, CLP: common lymphoid progenitor, GMP: granulocyte-macrophage progenitor, MEP: megakaryocyte-erythroid progenitor, pre-pro-B: pre-progenitor $B$, pro-B: progenitor $B$, pre-B: precursor B. Each symbol=one mouse. The horizontal line indicates the median of the result. *, $\mathrm{p}<0.05 ;{ }^{* *}, \mathrm{p}<0.01 ;{ }^{* * *}, \mathrm{p}<0.001$ (two-tailed Student's $t$-test).

Fig.6 UBXN3B maintains BLNK protein level and pre-BCR signaling. a) qRT-PCR quantification of gene expression in bone marrow B lineage subsets of specific pathogen-free littermates. Pre-pro-B: pre-progenitor B, pro-B: progenitor B, pre-B: precursor B, Neu: neutrophil, Mono: monocyte. b) The cellularity of surface Vpreb1+ cells and mean fluorescence intensity (MFI). Each symbol=one mouse. The horizontal line indicates the median of the result. *, $\mathrm{p}<0.05$; ${ }^{* *}, \mathrm{p}<0.01 ;{ }^{* *}, \mathrm{p}<0.001$ (two-tailed Student's t-test). c) The Uniform Manifold Approximation and Projection (UMAP) of surrogate light chain (SLC) gene expression by scRNA-seq. Cells in the oval express a high level of SLC (SLC ${ }^{\text {hi }}$, while cells in the rectangle express a low level of 
826 SLC $\left(S L C^{10}\right)$. d) The most significant pathways for the down-regulated genes in $U b x n 3 b^{-/-} \mathrm{SLC}^{\text {hi }}$

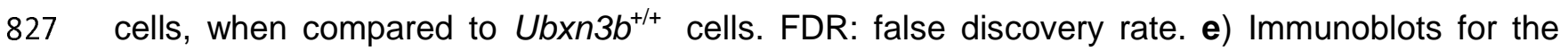

828 indicated proteins in bone marrow B fractions. L: large. f) The violin plots of Blnk transcript

829 levels by scRNAseq in bone marrow SLC ${ }^{\text {hi }}$ and SLC ${ }^{\text {lo }} \mathrm{B}$ cells. $\mathrm{g}$ ) The ratio of $\Delta \mathrm{F}$ (the difference

830 of calcium load between any a given time after anti-lgM $\mu \mathrm{H}$ treatment and time point zero F0) to

831 F0. Each dot represents the ratio of mean $\triangle F / F 0$ of all the cells recorded at a given time (every

832 second). Below each chart is the immunoblot of BLNK. $\mathbf{c - g}$ ) represent the results from three

833 mice.

834

835 Supplemental Materials

836 Supplemental Figs. 1-9.

837 Supplemental Table 1-4.

838 Supplemental Movie 1: SARS-CoV-2-infected Ubxn3b $b^{+/+}$mice on day 2 after infection.

839 Supplemental Movie 2: SARS-CoV-2-infected Ubxn3- $b^{-/-}$mice on day 2 after infection.

840 
Fig.1

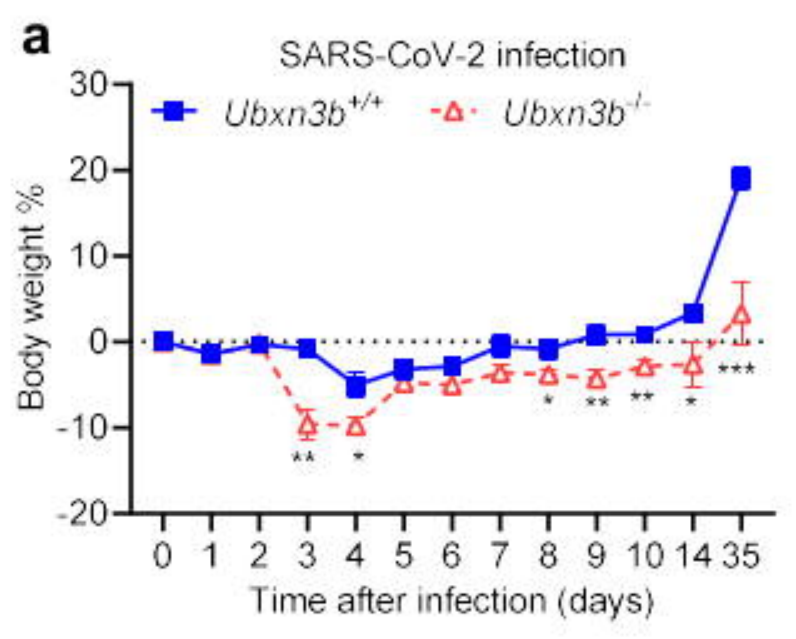

C

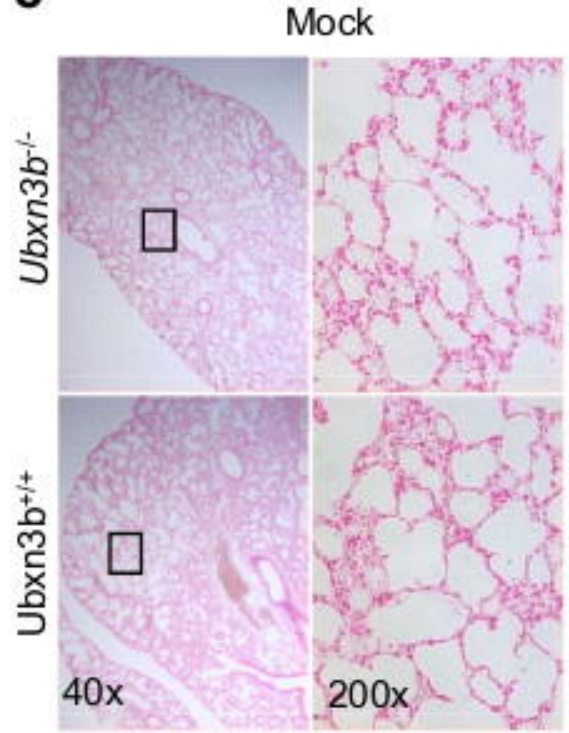

b

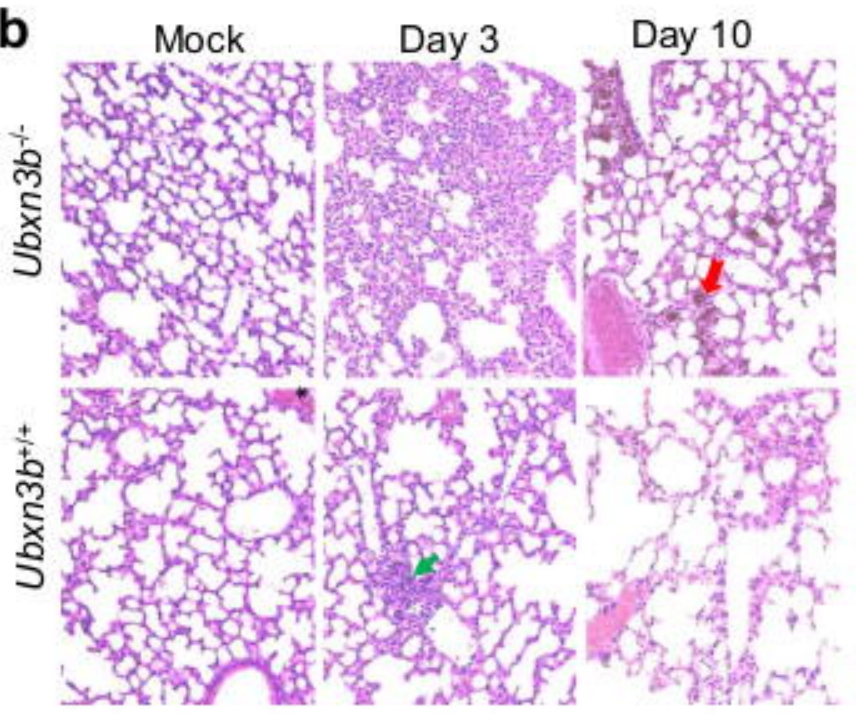

d Lung hemosiderosis

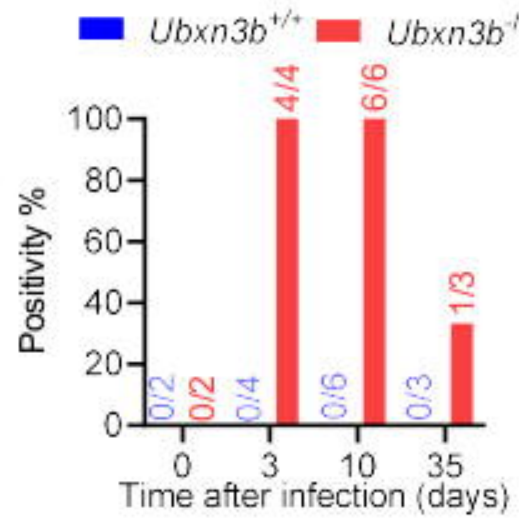

e

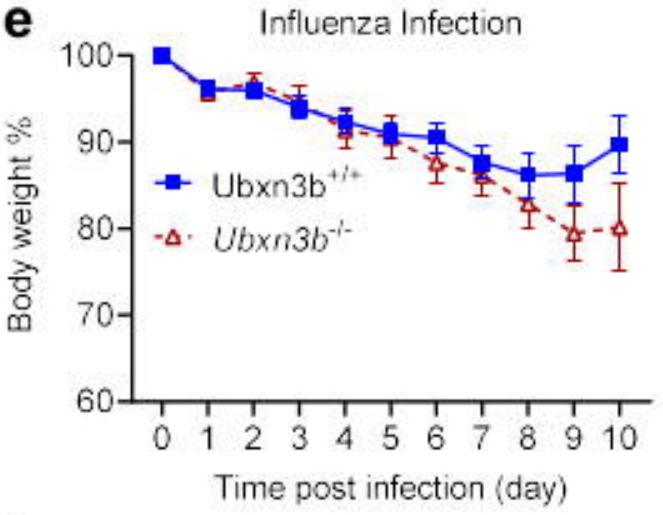

f

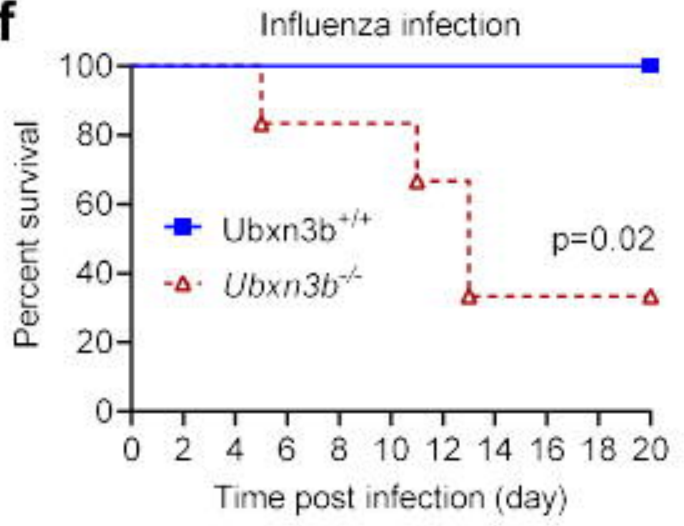


Fig.2
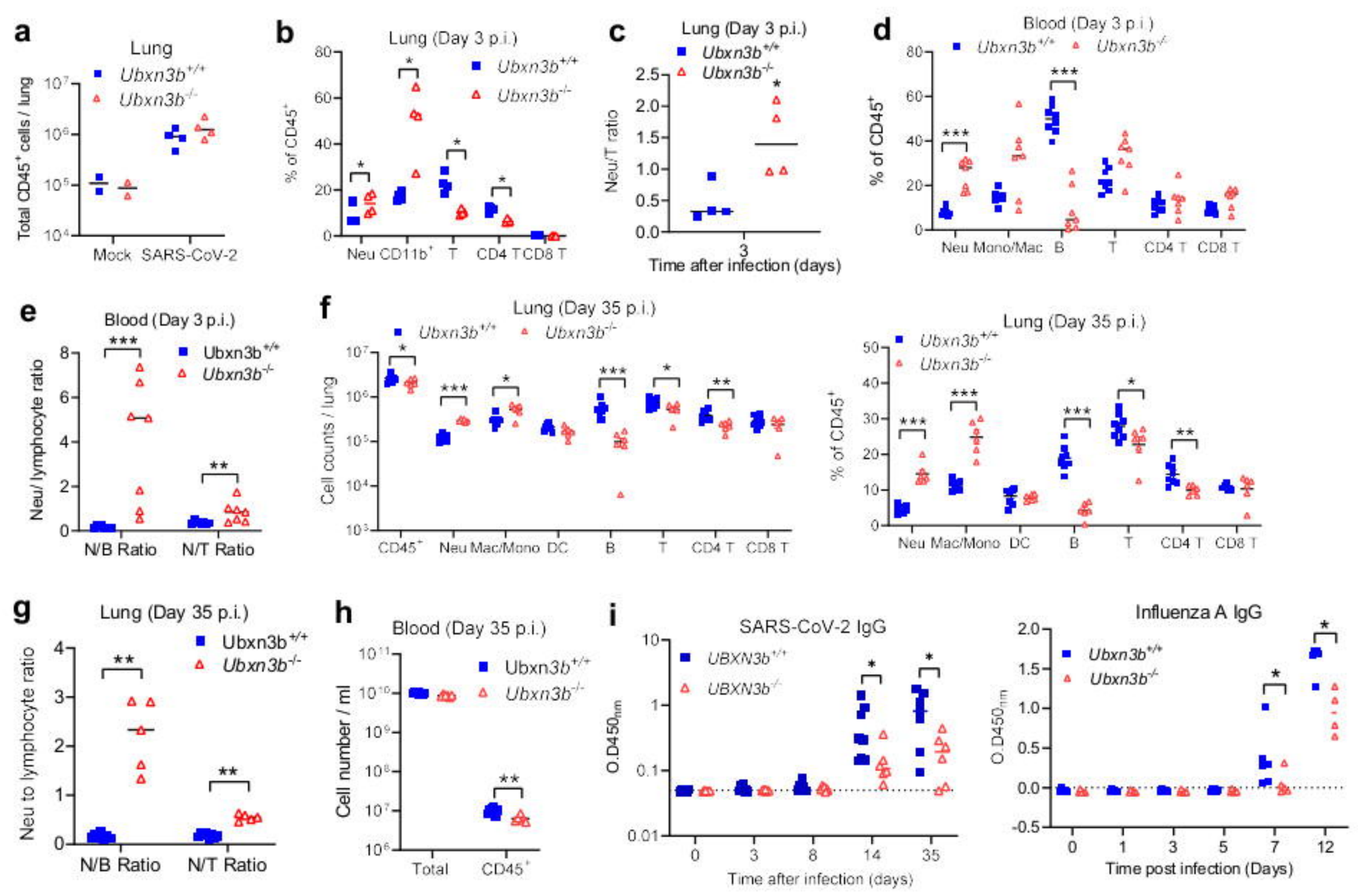
Fig. 3
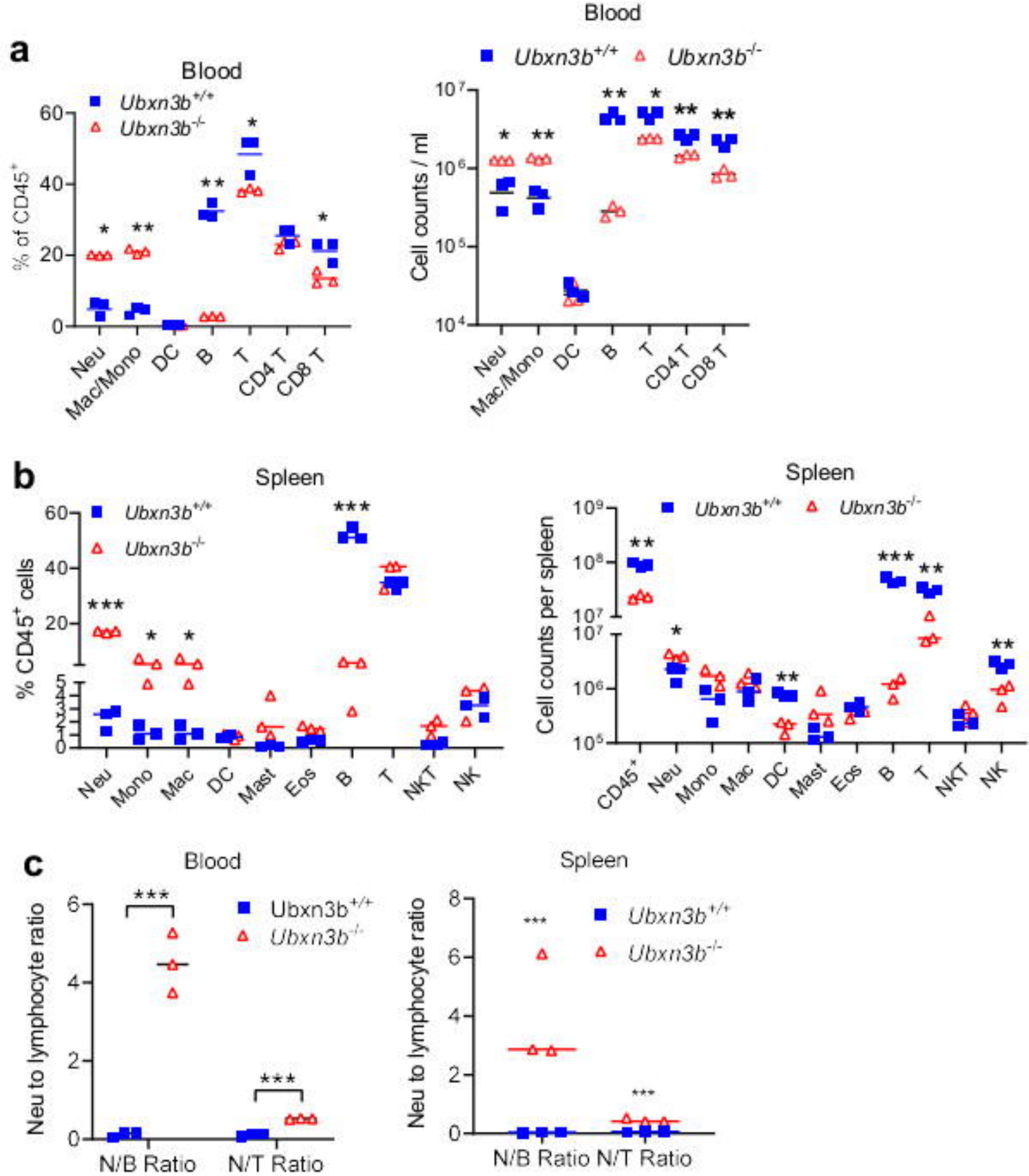
Fig.4
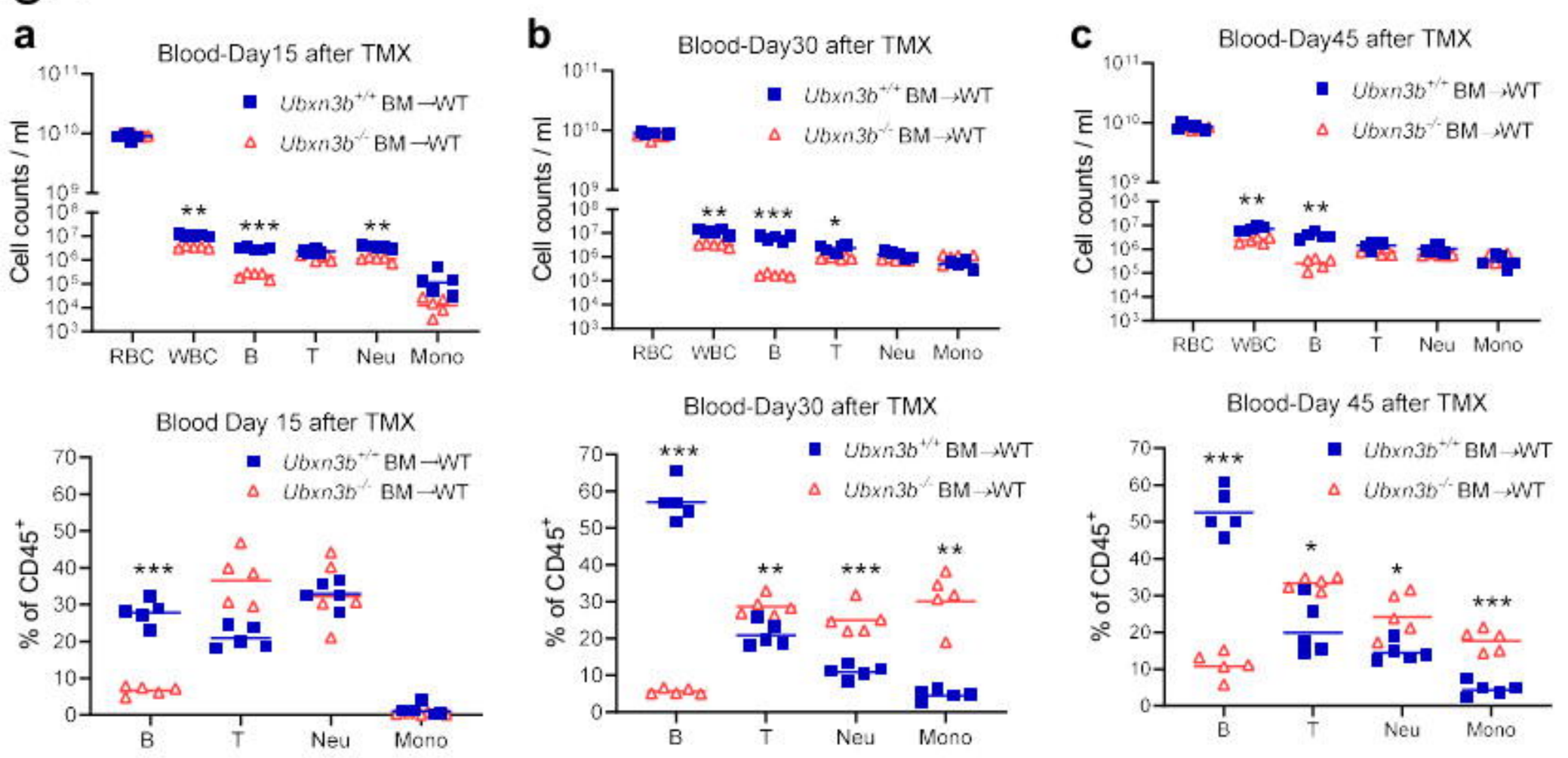

d

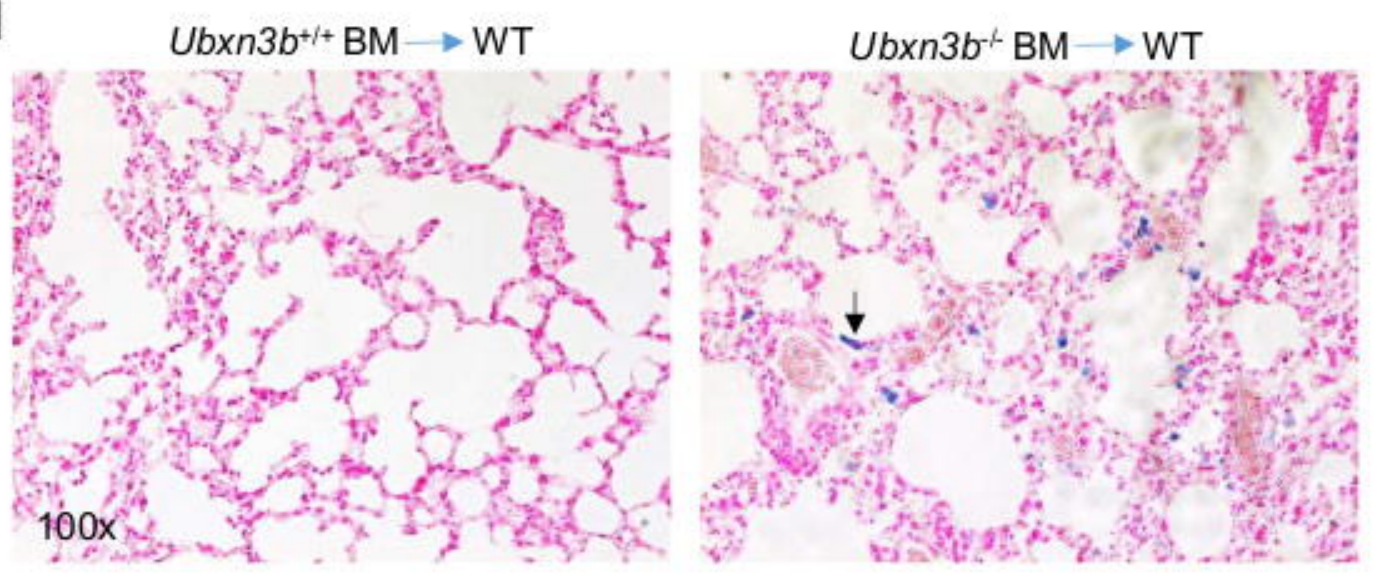


Fig.5

a

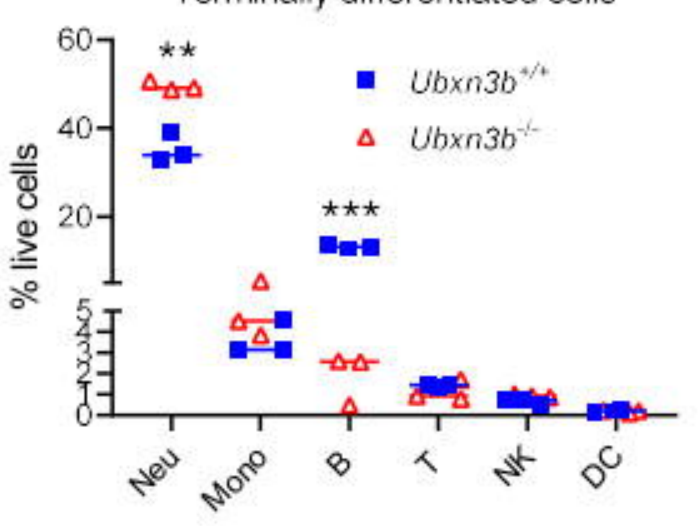

b

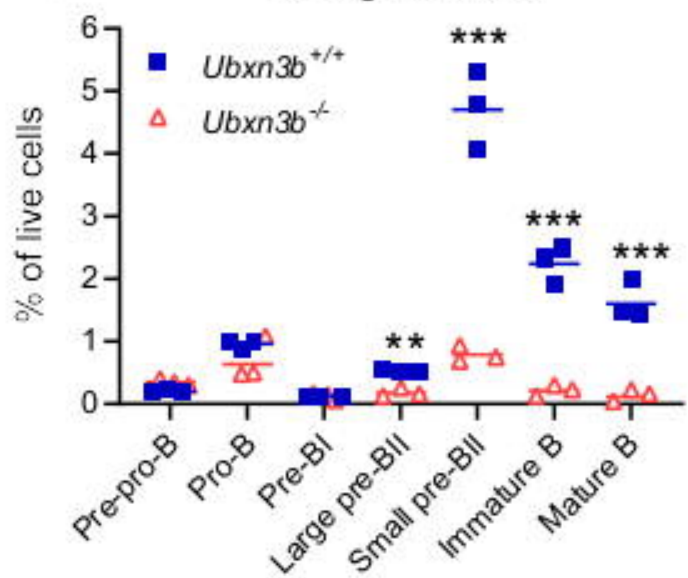

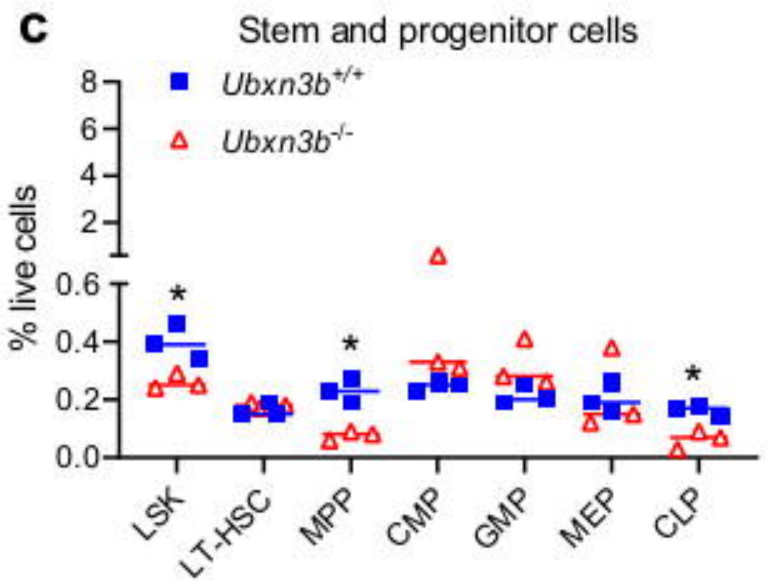

B-linegae subset

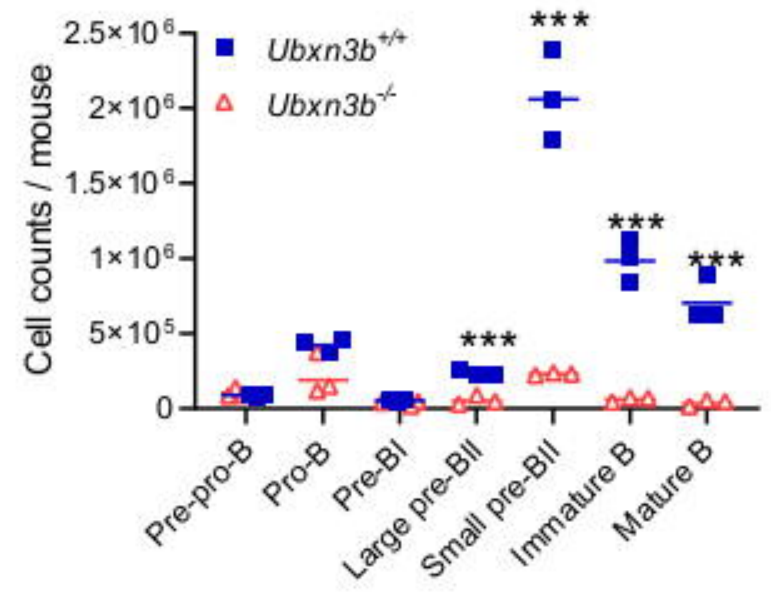


Fig. $6^{\text {bioRxiv preprint doi: https://doi.org/10.1101/2021.03.04.433919; this version posted August 24, 2021. The copyright holder for this preprint }}$ (which was not certified by peer review) is the author/funder. All rights reserved. No reuse allowed without permission.
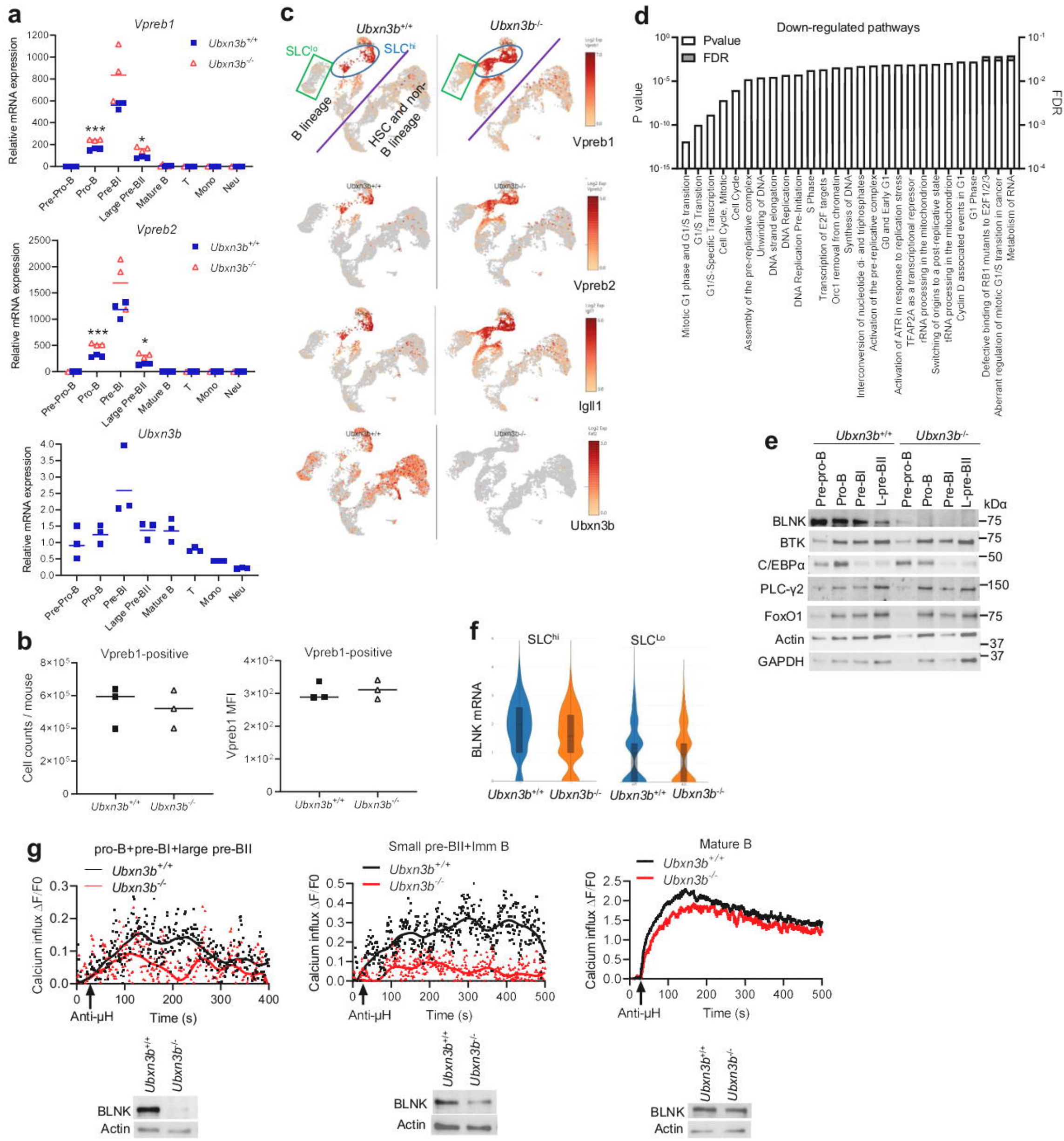